\title{
Effect of Free Stream Turbulence and Other Vortical Disturbances on a Laminar Boundary Layer
}

\author{
By S. J. Leib ${ }^{1}$, David พ. Wundrow ${ }^{1}$, and M. E. Goldstein ${ }^{2}$ \\ ${ }^{1}$ NYMA Inc. Lewis Research Center Group, Cleveland, Ohio 44135, USA \\ ${ }^{2}$ National Aeronautics and Space Administration, \\ Lewis Research Center, Cleveland, Ohio 44135, USA
}

\begin{abstract}
This paper is concerned with the effect of free-stream turbulence on the pretransitional flat-plate boundary layer. It is assumed that either the turbulence Reynolds number or the downstream distance for both) is small enough so that the flow can be linearized. The dominant disturbances in the boundary layer, which are of the Klebanoff type, are governed by the linearized unsteady boundaryregion equations, i.e., the Navier stokes equations with the streamwise derivatives neglected in the viscous and pressure-gradient terms. The turbulence is represented as a superposition of vortical free-stream Fourier modes, and the corresponding individual Fourier component solutions to the boundary-region equations are obtained numerically. The results are then superposed to compute the root mean square of the fluctuating streamwise velocity in the boundary layer produced by the actual free-stream turbulence. The calculated boundary-layer disturbances are in good quantitative agreement with the experimentally observed Klebanoff modes when strong low-frequency anisotropic effects are included in the free-stream turbulence spectrum. We discuss some additional effects that may need to be accounted for in order to obtain a complete description of the Klebanoff modes.
\end{abstract}




\section{Introduction}

This paper is concerned with the effects of vortical free-stream disturbances on transition to turbulence in flat-plate boundary layers. Weak free-stream turbulence in an otherwise uniform stream is probably the most important example of this type of disturbance, and Dryden (1936) and Taylor (1939) were the first to study its effects on the flat-plate boundary layer. They showed that the resulting streamwise velocity fluctuations were of very low frequency and reached amplitudes that were several times larger than those in the free-stream.

However, most of the early experiments were conducted at very low free-stream turbulence levels in order to confirm the existence of TS waves, and the Dryden-Taylor observations did not receive much attention until Klebanoff carried out his $1 \subseteq 71$ experiments--which is unfortunate since the free-stream turbulence level is usually quite high in both technological and naturally occurring flows. In addition to reproducing the earlier findings, Klebancff (1971) demonstrated that the disturbances grew more or less linearly with the boundarylayer thickness and that they are quite narrow in spanwise extent. Klebanoff (1971) referred to these disturbances as breathing modes, because, as noted earlier by Taylor (1939), they appeared to correspond to a thickening and thinning of the boundary layer. However, Kendall (1991) renamed them Klebancff modes, and that name seems to have taken hold even though they are not modes in the strict 
mathematical sense (i.e., they are not eigen-solutions of an appropriate differential equation) .

More recent experimental studies of this phenomena were carried out by Arnal and Juillen (1978), Kosorygin et al. (1982), Kendall (1985, 1991), Suder, O'Brien, and Reshotko (1988), Blair (1992), Roach and Brierley (1992), Westin et al. (1994), and Watmuff (1997). The most recent of these acknowledge the importance of carefully controlling and documenting the free-stream disturbance environment, but, as will be shown below, much remains to be done in this regard. Westin et al. (1994) collected results from a number of these experiments and showed that the root mean square ( $\mathrm{rms}$ ) of the streamwise velocity fluctuation initially grows like the square root of the distance from the leading edge of the plate, as observed by Klebanoff, but the actual growth rates differ from experiment to experiment even when the amplitudes are normalized by the free-stream turbulence level. Moreover, at least some of the data exhibits growth rates that decrease with increasing downstream distance, and in some cases there is a sudden increase in amplitude, presumably signaling the onset of transition.

There are many theoretical and numerical studies of the effect of small free-stream disturbances on flat-plate boundary layers, but only a few of these directly relate to the generation and growth of Klebanoff modes by free-stream turbulence. 
Motivated by the experimental findings of Bradshaw (1965), who suggested that the observed spanwise variations in shear stress on his plate could be traced to nonuniformities produced by the screens in the settling chamber, Crow (1966) carried out a linear analysis of the boundary-layer flow due to a small spanwise distortion of an otherwise uniform free-stream velocity. Goldstein, Leib and Cowley, (1992) and Goldstein and Leib (1993) considered the nonlinear boundary-layer flow due, respectively, to steady normal and streamwise vorticity distortions to an otherwise uniform upstream flow.

Bertolotti (1997) used the parabolized stab:lity equations to numerically compute the disturbance velocity due to steady and lowfrequency, single-Fourier-component, free-stream modes and compared the results with the experimental data of westin et al. (1994) and some recent unpublished data of Kendall. However, he was only able to obtain agreement with experiment by representing the free-stream turbulence by a single Fourier mode whose amplitude was chosen to fit the experimental data.

The actual free-stream turbulence is, of course, broadband and is therefore best represented as a superposition of Fourier modes. This superposition can only be carried out within the context of a linear analysis, which provides considerable motivation for extending the range of application of the linear theory as much as possible-especially since the only alternative appears to be the use of a full numerical simulation. 
Scaling of the governing equations shows (Goldstein et al., 1992; Goldstein and Leib, 1993; and Goldstein and Wundrow, 1997) that nonlinear effects become important when the scaled streamwise distance $\varepsilon * / L^{*}$ is order-one, where epsilon is a measure of the free-stream turbulence level and $L^{*}$ is its characteristic length scale. Fluctuating streamwise velocity measurements were taken by Westin et al. (1994) for two free-stream turbulence levels at distances from the leading edge ranging from $100 \mathrm{~mm}$ to $1 \mathrm{~m}$. At their higher turbulence level, $\varepsilon=.015$. Westin et al. (1994) estimate the transverse integral scale of the free-stream turbulence in their experiment to be between 7 and $10 \mathrm{~mm}$ (see page 203 of their paper). Using this length scale for $L^{*}$, and a value of $8 \mathrm{~mm}$, we find that the measurement points of Westin et al. (1994) lie within the range of $\varepsilon x^{*} / L^{*}$ values between about 0.2 to 2.0 . These estimates, which are in sharp disagreement with those made by Bertolotti (1997), suggest that, while linear theory should be able to describe the initial stages of the Klebanoff mode evolution, nonlinear effects are likely to be important further downstream.

Gulyaev et al. (1989) used solutions of the linearized, unsteady boundary-layer equations to describe the evolution of Klebanoff modes. They showed that the boundary-layer fluctuations are driven by two independent components of the free stream motion; a two-dimensional component, which is relatively benign, and a three-dimensional one that exhibits significant streamwise growth and therefore provides the dominant contribution to the rms streamwise velocity fluctuations. However, as even Gulyaev et al. (1989) point out, the Klebanoff modes 
cannot actually be described by the Prandtl boundary-layer equations because the spanwise length scales of these modes are usually of the same order as the local boundary-layer thickness at the streamwise location where most of the experimental measurements are taken.

In this paper, we carry out a systematic asymptotic analysis of the effect of vortical free-stream disturbances on a laminar flat-plate boundary layer. We assume that the turbulence Reynolds number is small enough so that the problem may be linearized. Our results show that the analysis of Gulyaev et al (1989) applies only at very small distances from the leading edge and that, in the region where experimental data are taken, the Klebanoff modes are governed by the unsteady boundary-region equations, i.e., the Navier-stokes equations with the streamwise derivatives neglected in the viscous and pressuregradient terms (Kemp 1951). The boundary-region equations are elliptic, rather than hyperbolic, in the crossflow plane, and this has a significant effect on the solutions.

Unlike the Bertolotti (1997) analysis, the upstream and far-field boundary conditions for the boundary-region equations solution result from strict asymptotic matching with a realistic free-stream turbulent flow.

The problem is formulated, and the appropriate scaling is developed in Section 2. In section 3, we consider the ilviscid flow above the boundary layer. The linearized unsteady boundary-layer region is discussed in Section 4 , and the region where the linearized unsteady 
boundary-region equations apply is discussed in section 5 . In Section 6 , the long transverse wavelength limit of the boundary-region solution is compared with the boundary-layer results, and it is shown that the spanwise ellipticity effects are much stronger than would be expected from order-of-magnitude considerations. In Section 7, we derive an expression for the streamwise velocity correlation function in the boundary layer by combining the individual Fourier-component solutions to the boundary-region equations with the upstream turbulence spectrum. The corresponding computations are presented in Section 8 , and the results are compared with data from some of the more recent experiments. The results of the analysis and computations are discussed in section 9.

\section{Formulation and scaling}

We consider the flow over an infinitely thin flat plate due to a stationary, homogeneous, grid-generated turbulence field. The relatively weak turbulence field that is imposed in most of the experiments is reasonably well-represented by a purely convected perturbation, say

$$
\mathbf{u}-\hat{i}=\varepsilon \mathbf{u}_{\infty}(x-t, y, z)
$$

of a nominally uniform mean flow, $U_{\infty}$, over a sufficiently small streamwise and transverse region, i.e., on a local basis (see Sections 3 and 6 below). Here $\mathbf{x}=x \hat{i}+y \hat{j}+z \hat{k}=\left\{x_{1}, x_{2}, x_{3}\right\}$ denote the cartesian coordinates normalized, along with all other lengths, by the transverse integral scale of the turbulence, $\Lambda$, with $x$ in the 
direction of the uniform mean flow, y normal to the plate, and $z$ along the span. The time, $t$, is made dimensionless with $\Lambda / U_{\infty}$, while the velocities and pressure are normalized with $U_{\infty}$ and $\rho U_{\infty}{ }^{2}$, respectively, where $\rho$ is the (constant) density. The smal parameter $\varepsilon$ is a measure of the turbulence intensity, and the scaled turbulence velocity $\mathbf{u}_{\infty}$ must satisfy the selnoidal condition

$$
\nabla \cdot \mathbf{u}_{\infty}=0,
$$

but can otherwise be specified arbitrarily as an upstream boundary condition.

Since the upstream turbulence is assumed to be stationary and homogeneous, it can be treated as a superposition of harmonic disturbances of the form

$$
\mathbf{u}_{\infty}=\hat{\mathbf{u}}^{\infty} e^{i\left(\mathbf{k} \cdot \mathbf{x}-k_{1} t\right)}
$$

with

$$
\hat{\mathbf{u}}^{\infty} \cdot \mathbf{k}=0
$$

where $\mathbf{k}=\left\{k_{1}, k_{2}, k_{3}\right\}$.

We suppose, at least initially, that the turbulence Reynolds number

$$
r_{t}=\varepsilon R \Lambda
$$

is $O(1)$ where

$$
\mathrm{R}_{\Lambda} \equiv \mathrm{U}_{\infty} \Lambda / \nu
$$

is the ordinary Reynolds number based on $\Lambda$. This corresponds to a kind of generic scaling from which the limits $r_{t} \rightarrow \infty$ and $r_{t} \rightarrow 0$ can be obtained as special cases. 
Then in the asymptotic limit $\varepsilon \rightarrow 0, R_{\Lambda} \rightarrow \infty$ with $r_{t}$ held fixed at 0 (1), the flow divides itself into four distinct asymptotic regions (see Figure 1).

The first of these is a primarily inviscid region of dimension $0(\Lambda)$ surrounding the leading edge, in which the motion can be treated as a linear perturbation about a uniform flow.

Beneath the linear region (1) is a region (denoted by (2)) where the flow is governed by the linearized unsteady laminar boundary-layer equations (Gulyaev et al., 1989; Goldstein, 1983). However, the mean boundary-layer thickness, $\Lambda \delta$, continues to grow with $x$, and this solution will become invalid at the downstream distance $\lambda^{2} R_{\Lambda} / \Lambda$ where $\Lambda \delta$ becomes of the order of the spanwise length scale $\lambda$ of the unsteady boundary-layer flow, which may, as we shall see, differ from the integral scale $\Lambda$. A new solution must then be obtained when

$$
x /\left(\frac{\lambda}{\Lambda}\right)^{2} R_{\Lambda}=\frac{\Lambda x^{*}}{\lambda^{2} R_{\Lambda}}=0(1)
$$

i.e., in region (3. The flow in this region is now fully threedimensional, because the spanwise derivatives in the viscous terms are no longer negligible compared with the normal derivatives. It will be 
shown subsequently that the unsteady components of the motion move out of the boundary layer with increasing downstream distance, and only the low-frequency components of the motion remain. The flow then evolves on the slow time scale

$$
\vec{t} \equiv t /\left(\frac{\lambda}{\Lambda}\right)^{2} R_{\Lambda}
$$

and is governed by the unsteady boundary-region equations (Kemp, 1951), which are just the Navier-Stokes equations with the streamwise derivatives neglected in the viscous and pressure-gradient terms.

Since the linearized unsteady boundary-laye:: solution grows linearly with $x$, it is easy to see from (2.7) that the solution in region (3) can be linearized about the undisturbed, Blasius solution when

$$
\varepsilon R_{\Lambda}\left(\frac{\lambda}{\Lambda}\right)=r_{t}\left(\frac{\lambda}{\Lambda}\right)<<1
$$

Finally, the flow in the large outer region (4) is in general nonlinear and corresponds to the usual equilibrium desay of grid-generated turbulence. It can, however, be linearized when the turbulence Reynolds number $r_{t}$ is much less than one and, more generally, will behave locally like a convected perturbation of the type (2.1) over distance $x_{L}$, for which

$$
\mathrm{x}_{\mathrm{L}} \ll 1 / \varepsilon=\mathrm{R}_{\Lambda} / \mathrm{r}_{\mathrm{t}}
$$


which means, in effect, that it can be linearized over such distances. These order-of-magnitude estimates are somewhat optimistic in that they do not properly account for the enhanced nonlinearity that can be produced by the smaller scale components of the turbulence. They should, however, be good enough to provide adequate estimates of the overall behavior of the flow.

Finally, we suppose that the upstream turbulence $\boldsymbol{\varepsilon} \mathbf{u}_{\infty}$ is specified at a distance $-x_{L}{ }^{\dagger}$, which is large compared to unity, but small compared to $\lambda^{2} \mathrm{R}_{\Lambda} / \Lambda^{2}$, i.e.,

$$
1 \ll-\mathrm{x}_{\mathrm{L}}^{\dagger} \ll\left(\frac{\lambda}{\Lambda}\right)^{2} R_{\Lambda} .
$$

The mean flow in this region will, in general, be nonuniform (see Section 3 and section 6 below), but it will vary slowly enough so that the upstream boundary condition (2.1) or (2.3) can still be specified on a local basis independently of that flow.

\section{The Linear Inviscid Solution}

The inviscid flow in region (1) can be determined by generalized rapiddistortion theory (Hunt, 1973; Goldstein, 1978). Since the problem is Iinear, we need only consider individual Fourier components (2.3) of the upstream distortion. 
The flow in this region, where $x$ and $y$ are order one, is given by Goldstein (1978) as

$$
\begin{gathered}
\mathbf{u}=\nabla \Phi+\varepsilon\left(\nabla \phi+\mathbf{u}^{(I)}\right), \\
p=-\frac{1}{2}|\nabla \Phi|^{2}-\varepsilon\left(\frac{\partial \phi}{\partial t}+\nabla \Phi \cdot \nabla \phi\right),
\end{gathered}
$$

where

$$
u_{i}^{(I)}=\mathbf{u}_{\infty}(\mathbf{X}-\hat{i} t) \cdot \frac{\partial \mathbf{X}}{\partial x_{i}}
$$

$\Phi$ is the known potential for the mean flow about the plate (including boundary-layer displacement effects), and

$$
\mathbf{X}=\{\Delta, \Psi, z\},
$$

with $\Psi$ denoting the mean-flow stream function and $\Delta$ the Lighthill (1956)-Darwin (1954) drift function.

For the infinitely thin flat plate of interest here, the complex meanflow potential is given, to the required order of accuracy, by

$$
\Phi+i \Psi=x+i y-i \beta \sqrt{\frac{2(x+i y)}{R_{\Lambda}}}
$$


(Van Dyke, 1964, pp. 132-135) while the corresponding drift function is

$$
\Delta=x+\operatorname{Re}\left[i \beta \sqrt{\frac{2(x+i y)}{R_{\Lambda}}}\right]
$$

where $\beta \sqrt{x}$ is the nondimensional displacement thickness determined by the Blasius solution with $\beta=1.217$ (Schlichting, 1955, page 106).

Finally, the perturbation potential $\phi$ is determined by the Poisson equation

$$
\nabla^{2} \phi=-\nabla \cdot \mathbf{u}^{(l)}
$$

subject to the boundary conditions that

$$
\begin{gathered}
\phi \rightarrow 0 \text { as } x \rightarrow-\infty, \\
\phi=0 \text { at } y=0, x<0, \\
\phi_{y}+u_{2}^{(I)}=\sqrt{\frac{2 x}{R_{\Lambda}}} k_{1} \bar{v}_{0}^{\infty} e^{i\left(k_{3} z-k_{1} t\right)} \text { at } y=0, x>0,
\end{gathered}
$$

where $\bar{v}_{0}^{\infty}$ is the scaled blowing velocity determined by the boundarylayer solution in region (2) and defined by (4.13) below. 
The order $1 / \sqrt{R_{\Lambda}}$ boundary-layer displacement effects in (3.5), (3.6), and (3.10) will need to be included in section 5.2 below in order to derive the upstream matching conditions for the flow in region (3). However, for the purposes of analyzing the flow in region (2), only the leading-order term is needed. Therefore, it follows from (3.3), $(3.4),(3.5)$, and $(3.6)$ that

$$
\mathbf{u}^{(I)}=\left\{\hat{u}_{1}^{\infty}, \hat{u}_{2}^{\infty}, \hat{u}_{3}^{\infty}\right\} e^{i\left(\mathbf{k} \cdot \mathbf{x}-k_{1} t\right)}+0\left(\frac{1}{\sqrt{R_{\Lambda}}}\right) .
$$

The leading-order solution of the boundary-value problem $(3.7)-(3.10)$ can be obtained using the Wiener-Hopf technique (e.g., Choudhari, 1996), but our interest is in the downstream form of the solution which can more easily be found by applicaticn of the method of variation of parameters. When the result, along with (3.11) and (3.5), is substituted into (3.1), we obtain

$$
\begin{gathered}
\mathbf{u}=\hat{i}+\cdots+\varepsilon e^{i\left[k_{1}(x-t)+k_{3} z\right]} \mathbf{u}^{(1)} \cdots \\
p=-\frac{1}{2}+\cdots
\end{gathered}
$$

where

$$
\begin{gathered}
u_{\sigma}^{(1)} \equiv \hat{u}_{\sigma}^{\infty} e^{i k_{2} y}+\frac{i k_{\sigma}}{\gamma} \hat{u}_{2}^{\infty} e^{-\eta y} \quad \text { for } \quad J=1,3 \\
u_{2}^{(1)} \equiv \hat{u}_{2}^{\infty}\left(e^{i k_{2} y}-e^{-\gamma y}\right)
\end{gathered}
$$


with $\gamma \equiv \sqrt{k_{1}^{2}+k_{2}^{2}}$.

The inviscid velocity at the surface of the plate $(y=0)$ is then given by

$$
\begin{aligned}
& u_{1}^{(1)}=\hat{u}_{1}^{\infty}+\frac{i k_{1}}{\gamma} \hat{u}_{2}^{\infty}, \\
& u_{3}^{(1)}=\hat{u}_{3}^{\infty}+\frac{i k_{3}}{\gamma} \hat{u}_{2}^{\infty} .
\end{aligned}
$$

\section{The Linear Boundary-Layer Solution}

Since the mean boundary layer is of the Blasius type, the solution in region (2) corresponding to the single Fourier-component inviscid solution $(3.12)-(3.15)$ is of the form

$$
\begin{gathered}
\mathbf{u}=\left\{F^{\prime}(\eta), \sqrt{\frac{1}{2 x R_{\Lambda}}}\left(\eta F^{\prime}-F\right), 0\right\} \\
+\varepsilon\left\{\bar{u}_{0}(\bar{x}, \eta), \sqrt{\frac{2 \bar{x} k_{1}}{R_{\Lambda}}} \bar{v}_{0}(\bar{x}, \eta), \bar{w}_{0}(\bar{x}, \eta)\right\} e^{i\left(k_{3} z-k_{1}\right)},
\end{gathered}
$$

with the Blasius function determined, in the usual way, by 


$$
F^{\prime \prime \prime}+F F^{\prime \prime}=0
$$

with $F(0)=0, F^{\prime}(0)=0, F \rightarrow \eta-\beta$ as $\eta \rightarrow \infty$,

$$
\eta=y \sqrt{\frac{R_{\Lambda}}{2 x}}=0(1)
$$

and

$$
\bar{x}=k_{1} x,
$$

is a scaled streamwise variable. The velocity perturbation is determined by the unsteady, linearized, three-dimensional, boundarylayer equations

$$
\begin{gathered}
-i \bar{u}_{0}+F^{\prime} \frac{\partial \bar{u}_{0}}{\partial \bar{x}}-\frac{F}{2 \bar{x}} \frac{\partial \bar{u}_{0}}{\partial \eta}-\frac{\bar{u}_{0}}{2 \bar{x}} \eta F^{\prime \prime}+\overline{\mathrm{v}}_{0} F^{\prime \prime}=\frac{1}{2 \bar{x}} \frac{\partial^{2} \bar{u}_{0}}{\partial \eta^{2}} \\
-i \bar{w}_{0}+F^{\prime} \frac{\partial \bar{w}_{0}}{\partial \bar{x}}-\frac{F}{2 \bar{x}} \frac{\partial \bar{w}_{0}}{\partial \eta}=\frac{1}{2 \bar{x}} \frac{\partial^{2} \bar{w}_{0}}{\partial \eta^{2}}, \\
\frac{\partial \bar{u}_{0}}{\partial \bar{x}}-\frac{\eta}{2 \bar{x}} \frac{\partial \bar{u}_{0}}{\partial \eta}+\frac{\partial \bar{v}_{0}}{\partial \eta}+i\left(\frac{k_{3}}{k_{1}}\right) \bar{w}_{0}=0,
\end{gathered}
$$

subject to the boundary conditions

$$
\bar{u}_{0}=\overline{\mathrm{v}}_{0}=\bar{w}_{0}=0 \text { at } \eta=0 \text {, }
$$

and that $\bar{u}_{0} e^{-i \bar{x}}$ and $\bar{w}_{0} e^{-i \bar{x}}$ match onto (3.16) ald (3.17) as $\eta \rightarrow \infty$. 
Since $k_{1}$ and $k_{3}$ only appear as multiplicative factors in (4.6), the boundary conditions (3.16) and (3.17) suggest that we divide up the solution in the following way (Gulyaev et al., 1989)

$$
\begin{aligned}
& \bar{u}_{0}=\left(\hat{u}_{1}^{\infty}+\frac{i k_{1}}{\gamma} \hat{u}_{2}^{\infty}\right) \bar{u}^{(0)}+i \frac{k_{3}}{k_{1}}\left(\hat{u}_{3}^{\infty}+\frac{i k_{3}}{\gamma} \hat{u}_{2}^{\infty}\right) \bar{u}, \\
& \bar{v}_{0}=\left(\hat{u}_{1}^{\infty}+\frac{i k_{1}}{\gamma} \hat{u}_{2}^{\infty}\right) \bar{v}^{(0)}+\frac{i k_{3}}{k_{1}}\left(\hat{u}_{3}^{\infty}+\frac{i k_{3}}{\gamma} \hat{u}_{2}^{\infty}\right) \overline{\mathrm{v}},
\end{aligned}
$$

and

$$
\bar{w}_{0}=\left(\hat{u}_{3}^{\infty}+\frac{i k_{3}}{\gamma} \hat{u}_{2}^{\infty}\right) \bar{w}
$$

Then $\{\bar{u}, \bar{v}, \bar{w}\}$ and $\left\{\bar{u}^{(0)}, \bar{v}^{(0)}, 0\right\}$ each satisfy the momentum equations (4.5), the latter satisfies the continuity equation (4.6), while the former satisfies

$$
\frac{\partial \bar{u}}{\partial \bar{x}}-\frac{\eta}{2 \bar{x}} \frac{\partial \bar{u}}{\partial \eta}+\frac{\partial \bar{v}}{\partial \eta}+\bar{w}=0
$$

Both components satisfy the no-slip condition (4.7) at the wall, and matching with (3.16) and (3.17) requires

$$
\left.\begin{array}{l}
\bar{u} \rightarrow 0, \quad \bar{w} \rightarrow e^{\bar{x}} \\
\bar{u}^{(0)} \rightarrow e^{\bar{x}}
\end{array}\right\} \quad \text { as } \eta \rightarrow \infty
$$


The solution of $(4.5)$ and (4.6) (or (4.11)) must satisfy appropriate upstream boundary conditions as $\bar{x} \rightarrow 0$. The time-dependent terms $-i \overline{u_{0}}$ and $-i \overline{w_{0}}$ drop out of (4.5) in this limit, and the relevant solutions are easily shown to be

$$
\left.\begin{array}{l}
\bar{u} \rightarrow \frac{\bar{x} \eta F^{\prime \prime}}{2}, \quad \overline{\mathrm{v}} \rightarrow \frac{1}{4}\left(\eta^{2} F^{\prime \prime}-3 \eta F^{\prime}-F\right), \quad \bar{w} \rightarrow F^{\prime} \\
\bar{u}^{(0)} \rightarrow \frac{1}{2}\left[\left(\eta F^{\prime}\right)^{\prime}+F^{\prime}\right], \quad \overline{\mathrm{v}}^{(0)} \rightarrow \frac{1}{4 \bar{x}}\left[\eta\left(\eta F^{\prime}\right)^{\prime}-F\right]
\end{array}\right\} \text { as } \bar{x} \rightarrow 0
$$

which shows that the streamwise velocity $\bar{u}^{(0)}$ remains bounded while $\bar{u}$ grows linearly with $\bar{x}$.

We shall make use of the fact that the blow:ng velocity, which appears in $(3.10)$, is defined by

$$
\overline{\mathrm{v}}_{0}^{\infty} \equiv \lim _{\eta \rightarrow \infty}\left[\overline{\mathrm{v}}_{0}+\left(\bar{u}_{0} \bar{x}+i \frac{k_{3}}{k_{1}} \bar{w}_{0}\right) r_{i}\right],
$$

and, in view of $(4.8)-(4.10)$ and $(4.13)$, behaves like

$$
\overline{\mathrm{v}}_{0}^{\infty} \sim \frac{\beta}{4}\left[\left(\hat{u}_{1}^{\infty}+\frac{i k_{1}}{\gamma} \hat{u}_{2}^{\infty}\right)\left(\frac{1}{\bar{x}}+\cdots\right)+i \frac{k_{3}}{k_{1}}\left(\hat{u}_{3}^{\infty}+\frac{i k_{3}}{\gamma} \hat{u}_{2}^{\infty}\right)(1+\cdots)\right]
$$

as $\bar{x} \rightarrow 0$. This result will be used in section 5.2 when the upstream boundary conditions for the solution in region (3) are considered. 
The solution in region (2) depends on the frequency parameter, $k_{1}$, only through the scaled streamwise variable $\bar{x}$. The limit $\bar{x} \rightarrow \infty$ may therefore be interpreted as either the high-frequency limit, $k_{1} \rightarrow \infty$, with $\mathrm{x}$ fixed or the downstream limit, $x \rightarrow \infty$, with $k_{1}$ fixed.

Similarly, the limit $\bar{x} \rightarrow 0$ may be interpreted as the low-frequency limit, $k_{1} \rightarrow 0$, with $x$ held fixed or the upstream limit, $x \rightarrow 0$, with $k_{1}$ fixed.

$\left\{\bar{u}^{(0)}, \bar{v}^{(0)}, 0\right\}$ has no crossflow component and is therefore twodimensional. The most recent numerical solutions for this quantity are given by Choudhari (1996). His results show that the disturbance velocity moves out of the boundary layer as $\bar{x}$ increases.

Our interest here is in the three-dimensional component, $\{\bar{u}, \bar{v}, \bar{w}\}$, whose streamwise velocity initially increases linearly with $\bar{x}$ at a rate which increases with increasing spanwise wave number. Before presenting numerical solutions for this component, we first consider its asymptotic solution as $\bar{x} \rightarrow \infty$. 


\subsection{Asymptotic Solution for $\bar{x} \rightarrow \infty$}

This solution is of the WKBJ form

$$
\{\bar{u}, \overline{\mathrm{v}}, \overline{\mathrm{w}}\}=\left\{\bar{U}(\eta, \bar{x}), \frac{\bar{V}(\eta, \bar{x})}{\sqrt{2 \bar{x}}}, \bar{W}(\eta, \bar{x})\right\} e^{i \bar{x}-\sqrt{2 \bar{x}} \Theta(\eta)} .
$$

in the region where $\eta=0(1)$.

Substituting this into the linearized boundary-layer equations and taking the limit as $\bar{x} \rightarrow \infty$ shows that

$$
\Theta^{\prime 2}=i\left(F^{\prime}-1\right)
$$

at lowest approximation, and that at next order that crossflow amplitude $\bar{W}$ is determined by

$$
\left(F^{\prime} \Theta-F \Theta^{\prime}\right) \bar{W}=2 \Theta^{\prime} \bar{W}^{\prime}+\Theta^{\prime \prime} \bar{W}^{\top}
$$

with a similar equation for $\bar{U}$.

Equation (4.18) is easily integrated to show that

$$
\bar{w}=\frac{c_{0}(\bar{x})}{\sqrt{\Theta^{\prime}}} e^{H(\eta)+i \bar{x}-\sqrt{2 \bar{x} \Theta}}
$$


where

$$
\Theta(\eta)=e^{-i \pi / 4} \int_{\eta}^{\infty} \sqrt{1-F^{\prime}} d \eta
$$

and

$$
H \equiv \frac{1}{2}\left(\ln F^{\prime \prime}+\int_{0}^{\eta} \frac{F^{\prime} \Theta}{\Theta^{\prime}} d \eta\right)
$$

which clearly shows that $\bar{w}$ decays exponentially fast as $\bar{x} \rightarrow \infty$ provided $\Theta=0(1)$. But $\Theta \rightarrow 0$ as $\eta \rightarrow \infty$, and the wKBJ approximation therefore breaks down, i.e. it has a turning point, when

$$
\sqrt{2 \bar{x}} \Theta=0(1)
$$

or in view of the fact that, $F \rightarrow \bar{\eta}+\frac{A}{\bar{\eta}^{2}} \exp \left(\frac{-\bar{\eta}^{2}}{2}\right)$, as $\bar{\eta}=\eta-\beta \rightarrow \infty$, with $A=0.331$ (Schlichting, 1955), when

$$
2 \bar{x} A=\frac{\bar{\eta}^{3}}{4} e^{\bar{\eta}^{2} / 2}
$$

A new solution, therefore, has to be obtained in this region, which we refer to as the edge layer. The relevant expansion for this region was first proposed by Gulyaev et al. (1989) and is developed here in more detail in Appendix A. 
While the solution (4.19) is exponentially small at $\eta=0$, it still does not vanish there. However, it can be canceled out at this point by adding a multiple of the additional solution corresponding to the other root of $(4.17)$. This amounts to replacing $\Theta(\eta)$ in $(4.17)$ by

$$
\Theta \rightarrow 2 \Theta(0)-\Theta(\eta)
$$

which also satisfies (4.17). This solution is exponentially smaller than (4.19) for all $\eta>0$ and is therefore negligible there. A similar procedure can be used for the other velocity components. But in doing this, use has to be made of an additional exact solution discovered by Lam and Rott. The result is again negligible compared with the primary solution for all $\eta>0$.

This analysis shows that the velocity fluct dations actually move out into the edge layer as $x \rightarrow \infty$ with $k_{1}$ fixed ard that the boundary-layer fluctuations at any given $\mathrm{x}$ will be dominated by the frequencies corresponding to $\bar{x}=k_{1} x=0(1)$.

\subsection{Numerical Solution}

Since the mean flow is two-dimensional, the spanwise momentum equation can be solved independently of the streamwise momentum and continuity 
equations. The latter two equations can be solved by decomposing the transverse velocity as follows:

$$
\bar{v}=\bar{v}^{(1)}+\bar{v}^{(2)}
$$

where

$$
\frac{\partial \bar{v}^{(2)}}{\partial \eta}+\bar{w}=0
$$

and

$$
\frac{\partial \bar{u}}{\partial \bar{x}}-\frac{\eta}{2 \bar{x}} \frac{2 \bar{u}}{2 \eta}+\frac{\partial \overline{\mathrm{v}}^{(1)}}{2 \eta}=0
$$

$\bar{v}^{(2)}$ can then be computed from (4.26), and $\bar{u}, \bar{v}^{(1)}$ from an inhomogeneous form of the two-dimensional, linearized, unsteady boundary-layer equations-once the spanwise velocity has been found.

A streamwise marching procedure, based on second-order central differences in $\eta$ and backward differences in $\bar{x}$, is used to obtain the solution at a desired streamwise station using the previously computed solutions upstream. Simpson integration is used to compute $\overline{\mathbf{v}}^{(2)}$. Two terms in the upstream expansion, $\bar{x} \rightarrow 0$, of which the first of (4.12) is the leading term, are used as the initial condition to start the procedure. 
Figures 2-4 are plots of the streamwise and spanwise velocity profiles computed from the boundary-layer equations. These results, like those obtained by Choudhari (1996), show that the disturbance velocity moves out of the boundary layer with increasing $\bar{x}$, but at a slower rate than the $\left(\bar{u}^{(0)}, \bar{v}^{(0)}\right)$ component, and that the streamise velocity exhibits a pronounced peak at an order-one value of $\eta$, which increases toward the boundary-layer edge as $\bar{x}$ increases.

As noted by Choudhari (1996), this streamwise velocity profile strongly resembles that of the Klebanoff modes generated by turbulence in the free-stream--even though these results correspond to a single Fourier component of the free-stream motion.

Figure 5 is a plot of the peak of the streanwise velocity and the associated transverse position as a function of $\bar{x}$. The asymptotic solutions shows that its spanwise location, say $\eta_{0}$, moves out of the boundary layer like $(\ln \bar{x})^{\frac{1}{2}}$ as $\bar{x} \rightarrow \infty$ (Brown and Stewartson, 1973).

These numerical results, together with the asymptotic solution, suggest that the streamwise velocity fluctuations will be dominated by their low-frequency components, $k_{1}<<1$, at the large downstream 
distances where $x>>1$. And since the actual physical solution is multiplied by the wave number ratio $k_{3} / k_{1}$, the small spanwise length scale components should exhibit the most rapid growth, which is consistent with the observations by Klebanoff and subsequent researchers.

Klebanoff found the spanwise wave length to be five times larger than the boundary-layer thickness, but in most, (if not all) of the more recent experiments (Kendall, 1985; Westin et al., 1994), the spanwise wave length was found to be nearly equal to the boundary-layer thickness, so that the relevant solutions cannot be determined by the linearized boundary-layer equations. This may, of course, only apply to the $\bar{u}, \overline{\mathrm{v}}, \bar{w}$ component of the solution; the $\bar{u}^{(0)}, \overline{\mathrm{v}}^{(0)}$ component is not subject to the short spanwise wave length selection process and may, therefore, continue to be governed by the linear boundary-layer equations until the boundary-layer thickness becomes $O(\Lambda)$. (See discussion in section 2.)

\section{The Linear Boundary-Region Equations}

As the low-frequency, small spanwise length-scale components of the unsteady motion grow downstream, and the boundary-layer thickness continues to increase, the classical boundary-layer equations 
eventually become invalid. This occurs wher $x \approx\left(\frac{\lambda}{\Lambda}\right)^{2} R_{\Lambda}$ (see eq. (2.7)), and since $\bar{x}=k_{1} x$ remains order one, this implies that $k_{1} R_{\Lambda}=O(1)$. Introducing the relevant component of the pressure,

$$
p=\frac{1}{2}+\cdots+\varepsilon i \kappa \sqrt{\frac{k_{1}}{R_{\Lambda}}}\left(\hat{u}_{3}^{\infty}+\frac{i k_{3}}{\gamma} \hat{u}_{2}^{\infty}\right) \bar{p} e^{i\left(k_{3} z-k_{1} l\right)}
$$

and the rest of the scalings in Section 4, into the linearized NavierStokes equations, we obtain

$$
\begin{gathered}
-i \bar{u}+F^{\prime} \frac{\partial \bar{u}}{\partial \bar{x}}-\frac{F}{2 \bar{x}} \frac{\partial \bar{u}}{\partial \eta}-\frac{\eta F^{\prime \prime}}{2 \bar{x}} \bar{u}+\overline{\mathrm{v}} F^{\prime \prime}=\frac{1}{2 \bar{x}} \bar{u}_{\eta \eta}-\kappa^{2} \bar{u} \\
-i \overline{\mathrm{v}}+F^{\prime} \frac{\partial \overline{\mathrm{v}}}{\partial \bar{x}}-\frac{F}{2 \bar{x}} \frac{\partial \overline{\mathrm{v}}}{\partial \eta}-\frac{\bar{u}}{(2 \bar{x})^{2}}\left[\eta\left(\eta F^{\prime}\right)^{\prime}-F\right] \\
+\frac{\overline{\mathrm{v}}}{2 \bar{x}}\left(\eta F^{\prime}\right)^{\prime}=-\frac{1}{2 \bar{x}} \frac{\partial \bar{p}}{\partial \eta}+\frac{1}{2 \bar{x}} \frac{\partial^{2} \overline{\mathrm{v}}}{\partial \eta^{2}}-\kappa^{2} \overline{\mathrm{v}} \\
-i \bar{w}+F^{\prime} \frac{\partial \bar{w}}{\partial \bar{x}}-\frac{F}{2 \bar{x}} \frac{\partial \bar{w}}{\partial \eta}=\kappa^{2} \bar{p}+\frac{1}{2 \bar{x}} \frac{\partial^{2} ; \bar{w}}{\partial \eta^{2}}-\kappa^{2} \bar{w} \\
\frac{\partial \bar{u}}{\partial \bar{x}}-\frac{\eta}{2 \bar{x}} \frac{\partial \bar{u}}{\partial \eta}+\frac{\partial \bar{v}}{\partial \eta}+\bar{w}=0
\end{gathered}
$$

in the limit $k_{1} / R_{\Lambda} \rightarrow 0$ with $\bar{x}, y=0(1)$, where we have put

$$
\kappa \equiv k_{3} / \sqrt{k_{1} R_{\Lambda}}=O(1)
$$

Following Kemp (1951) and Davis and Rubin (1980), we refer to (5.2)(5.5) as the linearized, unsteady boundary-region equations. They are 
simply the linearized Navier-Stokes equations with the streamwise derivatives neglected in the viscous and pressure-gradient terms, and, as we have shown, they correspond to a rational asymptotic limiting form of the Navier-stokes equations.

The boundary-region equations must be solved subject to appropriate far-field and upstream boundary conditions which, despite the similar nature of the equations, are rather more intricate than those for the boundary-layer equations. We derive the edge conditions for $\eta \rightarrow \infty$ by first considering the flow above region (3). The large- $\eta$ solution of (5.2)-(5.5) that matches with this solution then provides the correct edge boundary condition. The upstream condition for $\bar{x} \rightarrow 0$ must now, in addition to being specified for $\eta=0(1)$, be provided over the larger region $y_{0}=\eta \sqrt{2 \bar{x}}=0(1)$ to account for the increased boundarylayer thickness in region (3).

We again need only consider a single Fourier component of the upstream distortion velocity (2.3), but, as noted in section 2, the mean boundary-layer displacement will now affect the solution for the perturbed flow at leading order. In the outer region described by $k_{1} x, k_{1} y=0(1)$, the velocity expands like

$$
\mathbf{u}=\left\{\frac{\partial \Psi}{\partial y},-\frac{\partial \Psi}{\partial x}, 0\right\}+\varepsilon e^{i\left(k_{3} z-k_{1} t\right)} \mathbf{u}^{(0)}+\cdots,
$$


where the mean-flow stream function $\Psi$ is given to the required order of accuracy by (3.5), and since the pressure fluctuation will vanish in this region, the perturbation velocity $\mathbf{u}^{(0)}=\mathbf{u}^{(0)}(\bar{x}, y)$ is determined by

$$
\left(-i+\frac{\partial}{\partial \bar{x}}-\frac{\partial \Psi}{\partial \bar{x}} \frac{\partial}{\partial y}-\frac{1}{k_{1} R_{\Lambda}} \frac{\partial^{2}}{\partial y^{2}}+\kappa^{2}\right) \mathbf{u}^{(0)}=0,
$$

which becomes

$$
\left(-i+\frac{\partial}{\partial \vec{x}}-\frac{1}{k_{1} R_{\Lambda}} \frac{\partial^{2}}{\partial \Psi^{2}}+\kappa^{2}\right) \mathbf{u}^{(0)}=O\left(k_{1}\right),
$$

upon introducing $\Psi$ as a new independent variable. The solution that satisfies the upstream boundary condition in (2.3) for $\left|k_{1} y\right| \ll\left|\bar{x}_{L}^{\dagger}\right|$, as well as the continuity equation, is

$$
\mathbf{u}^{(0)}=\hat{\mathbf{u}}^{\infty} e^{i\left(\bar{x}+k_{2}^{\Psi} \Psi\right)-\left(x^{2}+\kappa_{2}^{2}\right)\left(\bar{x}-\bar{x}_{L}^{\dagger}\right)}
$$

where $\bar{x}_{L}^{\dagger} \equiv k_{1} x_{L}^{\dagger}$ and

$$
\kappa_{2} \equiv k_{2} / \sqrt{\kappa_{1} R_{\Lambda}}
$$

is a scaled transverse wave number. It follows from (3.5) and (A-1) that

$$
\Psi \rightarrow \frac{y^{(0)}}{\sqrt{k_{1} R_{\Lambda}}} \text { as } \quad k_{1} y \rightarrow 0, \bar{x}>0
$$

and therefore that 


$$
\mathbf{u}^{(0)} \rightarrow \hat{\mathbf{u}}^{\infty} e^{i\left(\bar{x}+\kappa_{2} y^{(0)}\right)-\left(\kappa^{2}+\kappa_{2}^{2}\right) \bar{x}}
$$

as region (3) is approached where

$$
y^{(0)} \equiv \sqrt{2 \bar{x}} \bar{\eta}
$$

use has been made of (2.11).

\subsection{Far-Field Boundary Conditions}

The results of the previous subsection can now be used to determine the outer-edge boundary conditions for the boundary-region equations. Using the large $\eta$ form of the Blasius solution in (5.1)-(5.4), and rewriting in terms of $y_{0}=\eta \sqrt{2 \bar{x}}$, yields

$$
\begin{gathered}
-i \bar{u}+\frac{\partial \bar{u}}{\partial \bar{x}}+\frac{\beta}{\sqrt{2 \bar{x}}} \frac{\partial \bar{u}}{\partial y_{0}}=\frac{\partial^{2} \bar{u}}{\partial y_{0}^{2}}-\kappa^{2} \bar{u} \\
-i \overline{\mathrm{v}}+\frac{\partial \overline{\mathrm{v}}}{\partial \bar{x}}+\frac{1}{2 \bar{x}} \overline{\mathrm{v}}-\frac{\beta}{(2 \bar{x})^{2}} \bar{u}+\frac{\beta}{\sqrt{2 \bar{x}}} \frac{\partial \overline{\mathrm{v}}}{\partial y_{0}}=\frac{-1}{\sqrt{2 \bar{x}}} \frac{\partial \bar{p}}{\partial y_{0}}+\frac{\partial^{2} \overline{\mathrm{v}}}{\partial y_{0}^{2}}-\kappa^{2} \overline{\mathrm{v}} \\
-i \bar{w}+\frac{\partial \bar{w}}{\partial \bar{x}}+\frac{\beta}{\sqrt{2 \bar{x}}} \frac{\partial \bar{w}}{\partial y_{0}}=\kappa^{2} \bar{p}+\frac{\partial^{2} \bar{w}}{\partial y_{0}^{2}}-\kappa^{2} \bar{w} \\
\frac{\partial \bar{u}}{\partial \bar{x}}+\sqrt{2 \bar{x}} \frac{\partial \overline{\mathrm{v}}}{\partial y_{0}}+\bar{w}=0
\end{gathered}
$$

The solution to $(5.15)-(5.18)$ that matches with the outer solution is 


$$
\begin{gathered}
\bar{u}=0, \\
\overline{\mathrm{v}}=\frac{i e^{i \bar{x}}}{\left(\kappa_{2}-i|\kappa|\right) \sqrt{2 \bar{x}}}\left\{e^{i \kappa_{2} y^{(0)}-\left(\kappa^{2}+\kappa_{2}^{2}\right) \bar{x}}-e^{-|\kappa| y^{(0)}}\right\}+\frac{|\kappa| e^{i \bar{x}}}{\sqrt{2 \bar{x}}} e^{-|\kappa| y^{(0)}} \int_{0}^{\bar{x}} g(\bar{x}) e^{-i \bar{x}} d \bar{x}, \\
\bar{w}=\frac{e^{i \bar{x}}}{\kappa_{2}-i|\kappa|}\left\{\kappa_{2} e^{i \kappa_{2} y^{(0)}-\left(\kappa^{2}+\kappa_{2}^{2}\right) \bar{x}}-i|\kappa| e^{-|\kappa| y^{(0)}}\right\}+\kappa^{2} e^{i \bar{x}} e^{-|\kappa| y^{(0)}} \int_{0}^{\bar{x}} g(\bar{x}) e^{-i \bar{x}} d \bar{x},
\end{gathered}
$$

and

$$
\bar{p}=g(\bar{x}) e^{-|x| y^{(0)}},
$$

where we have used (2.4) with $k_{1}<<k_{2}, k_{3}$.

Equations (5.19)-(5.22) then provide the form of the far-field boundary conditions to be imposed on the boundary-region equations. The function $g(\bar{x})$ is, at this point, unknown, but its behavior as $\bar{x} \rightarrow 0$ will be determined in the next subsection by matching (5.22) with the solution in region (1).

\subsection{Upstream Boundary Conditions}

Since the boundary-region problem describes the evolution of the lowfrequency Fourier components downstream of the initial boundary-layer stage, it follows that the appropriate upstream matching conditions 
for this problem are given by the low-frequency downstream limit of the solutions in regions (1) and (2) (i.e., the limit as $x \rightarrow \infty$ with $\bar{x} \ll 1$ ).

The leading-order terms in the low-frequency, downstream limit of the velocity fluctuations in region (1) are arrived at by a straightforward reexpansion of $(3.12),(3.14)$, and (3.15) using $k_{1}<<k_{2}, k_{3}$ along with (2.4). Obtaining the leading-order term for the pressure fluctuation, however, requires (as noted in section 2) accounting for the boundarylayer displacement effects. This can now be done by using (3.3)(3.6), (4.13), and solving $(3.7)-(3.10)$ to order $1 / \sqrt{R_{\Lambda}}$. Reexpanding the result for $k_{1} \ll k_{2}, k_{3}, x \gg 1$ leads to

$$
\phi \sim \frac{1}{\left|k_{3}\right|} \hat{u}_{2}^{\infty}\left[1-i\left(3 k_{2}+i\left|k_{3}\right|\right) \frac{\beta}{4} \sqrt{\frac{2 x}{R_{\Lambda}}}+\cdots\right] e^{-\left|k_{3}\right| y+i\left[k_{1}(x-t)+k_{3} z\right]}
$$

It then follows from $(3.2),(3.5),(3.12),(3.14)$, and (3.15) that

$$
\begin{gathered}
\bar{u} \rightarrow 0, \\
\overline{\mathrm{v}} \rightarrow \frac{i}{\left(\kappa_{2}-i|\kappa|\right) \sqrt{2 \bar{x}}}\left[e^{i \kappa_{2} y_{0}}-e^{-|\kappa| y_{0}}\right], \\
\bar{w} \rightarrow \frac{1}{\left(\kappa_{2}-i|\kappa|\right)}\left[\kappa_{2} e^{i \kappa_{2} y_{0}}-i|\kappa| e^{-|\kappa| y_{0}}\right], \\
\bar{p} \rightarrow-\frac{3 \beta}{4|\kappa|} \frac{1}{\sqrt{2 \bar{x}}} e^{-|\kappa| y_{0}},
\end{gathered}
$$


as $\bar{x} \rightarrow 0$ with $y_{0}=\eta \sqrt{2 \bar{x}}$ held fixed. Comparison of equations (5.22) and (5.27) gives the leading order behavior of $g(\bar{x})$ for $\bar{x} \rightarrow 0$.

This completes the specification of the boundary-region problem. In the next subsection, we describe the numerical method used to obtain solutions.

\subsection{Numerical Solution}

The finite difference approximation for the boundary-region equations is essentially the same as that used for the boundary-layer equations, except that it was necessary to compute the pressure, absent in the boundary-layer computations, on a grid stagcered in the transverse direction relative to that for the velocity components. The treatment of the initial and edge boundary conditions, however, is quite different.

As already mentioned, the upstream boundary conditions must now be provided over the region $y_{0}=0(1)$, as well as for $\eta=0(1)$. A composite solution constructed from the solutions for $\bar{x} \rightarrow 0$ with $\eta=0(1),(4.12)$, and $y_{0}=0(1),(5.24)-(5.27)$, was used as the 
starting condition, and it was found that two terms in the upstream expansion, $\bar{x} \rightarrow 0$, were required to successfully start the procedure.

The edge boundary conditions (5.19)-(5.22) involve the unknown function, $g(\bar{x})$, induced by the pressure in the outer region. This unknown function was eliminated by imposing the mixed boundary conditions

$$
\begin{gathered}
\bar{u} \rightarrow 0, \\
\frac{\partial \bar{v}}{\partial \eta}+|\kappa| \sqrt{2 \bar{x}} \overline{\mathrm{v}} \rightarrow-e^{i\left(\bar{x}+\kappa_{2} \sqrt{2 \bar{x} \bar{\eta})} e^{-\left(\kappa^{2}+\kappa_{2}^{2}\right) \bar{x}}\right.} \\
\frac{\partial \bar{w}}{\partial \eta}+|\kappa| \sqrt{2 \bar{x}} \bar{w} \rightarrow i \kappa_{2} \sqrt{2 \bar{x}} e^{i\left(\bar{x}+\kappa_{2} \sqrt{2 \bar{x}} \bar{\eta}\right)} e^{-\left(\kappa^{2}+\kappa_{2}^{2}\right) \bar{x}}
\end{gathered}
$$

and

$$
\frac{\partial \bar{p}}{\partial \eta}+|\kappa| \sqrt{2 \bar{x}} \bar{p} \rightarrow 0
$$

as $\eta \rightarrow \infty$.

Second-order finite differences were used in these boundary conditions, and a block tri-diagonal linear system of equations is obtained, which was solved using a standard sparse system algorithm. All the equations must be solved simultaneously in this case, because of the coupling through the pressure. 
Figures 6 and 7 show profiles of the magnitudes of streamwise and spanwise perturbation velocity profiles at various values of $\bar{x}$ computed from the boundary-region equations with $\mathrm{k}=1, \kappa_{2}=-1$. The streamwise velocity profiles look similar to the corresponding boundary-layer profiles, but the strong spanwise ellipticity effects cause the peak level, which initially increases linearly with $\bar{x}$, to rapidly decrease to zero. The spanwise velocity profiles are quite different from the boundary-layer profiles (see figures 2-4) due to the matching requirements of (5.30).

The initial linear growth and subsequent desrease of the peak in the streamwise perturbation velocity profile is more clearly shown in Figure 8, which is a plot of $|\bar{u}|$ as a function of $\bar{x}$ at $\eta=1.64$, where the peak in the profiles occurs.

Results from additional computations show that the peak of the $|\bar{u}|$ profiles reaches a maximum and then decreases to zero very rapidly for larger values of $\kappa$ and (absolute value of) $\kappa_{2}$; while at smaller values, the initial growth and ultimate decay are much more gradual.

The linearized unsteady boundary-region equations possess a similarity solution of the form 


$$
\begin{aligned}
& \bar{u}=\frac{1}{\kappa^{2}} \hat{u}\left(\kappa^{2} \bar{x}, \eta ; \frac{\kappa_{2}}{\kappa}\right), \\
& \bar{v}=\hat{v}\left(\kappa^{2} \bar{x}, \eta ; \frac{\kappa_{2}}{\kappa}\right), \\
& \bar{w}=\hat{w}\left(\kappa^{2} \bar{x}, \eta ; \frac{\kappa_{2}}{\kappa}\right),
\end{aligned}
$$

in the limit as $\kappa \rightarrow \infty$ with $\frac{\kappa_{2}}{\kappa}=0(1)$, where $\hat{u}, \hat{\mathrm{v}}$, and $\hat{\mathrm{w}}$ are now determined by the linearized steady boundary-region equations.

Figure $9 a$ is a plot of $\kappa^{2} \bar{u}$ vs. $\kappa^{2} \bar{x}$ at a fixed $\frac{\kappa_{2}}{\kappa}=-1, \eta=1.64$ for various values of $k \geq 1$. These results clearly collapse on a single curve for $\kappa \geq 2$, and even the $\kappa=1$ result is not too far from this curve. In Figure 9b, we have plotted the same results vs. $\kappa \sqrt{\bar{x}}$. Notice that, except for a small region near the origin, the steady solution increases linearly with $\kappa \sqrt{\bar{x}}$ (and therefore with the boundary-layer thickness) up to $x \sqrt{\bar{x}}$ of about 0.5 . This might lead one to suppose that the Klebanoff modes can be represented as a single steady mode (Bertolotti, 1997), but, as we shall see, the unsteady (but low frequency) solutions make the dominant contribution to the rms of the streamwise velocity fluctuations in the boundary layer. 
On the other hand, the boundary-region equations reduce to the boundary-layer equations in the limit as $k \rightarrow 0$. Figure 10 shows plots of $|\bar{u}|$ vs. $\bar{x}$ at fixed $\frac{\kappa_{2}}{\kappa}=-1$, and $\eta$ again equal to 1.64 , at various values of $k \leq .1$. The dashed curve denotes the solution calculated from the boundary-layer equations. The results show that solutions to the boundary-region equations approach the boundary-layer results very slowly as $k \rightarrow 0$ so that the linearized unsteady boundary-layer solution used by Gulyaev et al. (1989) to calculate the streamwise velocity fluctuations is only valid for very small spanwise wave numbers. This shows that the full linearized unsteady boundary-region solutions must be used to describe the experimental results in which the spanwise wave length is invariably of the order of the boundarylayer thickness.

Figure 11 is a plot of profiles of the streamwise velocity $|\bar{u}| /\left|\bar{u}_{\max }\right|$ for various values of $\bar{x}$, with $\kappa=1, \kappa_{2}=-1$. The results clearly come close to collapsing on a single curve. The dashed curve is the normalized mode shape $\eta F^{\prime \prime}$ that appears in the upstream boundary condition (4.12). This is the mode shape originally used by Taylor (1939), and later by Klebanoff (1971), to fit their experimental data.

We will use the computed solutions of the boundary-region equations to evaluate the rms of the streamwise velocity perturbation in the 
boundary layer due to a broadband turbulent flow. But first, we will examine, in more detail, the long wavelength limit of the boundaryregion equations, since the slow convergence to the boundary-layer solution has such a dramatic effect on our results.

\section{Long Wavelength Limit of Linear Boundary-Region Equations}

The linearized, unsteady boundary-region equations (5.2)-(5.5) reduce to the linearized unsteady boundary-layer equations when $\kappa_{,} \kappa_{2} \rightarrow 0$, i.e., in the long spanwise wavelength limit. Results from our numerical computations of the previous section show that this limit is approached very slowly.

In the long spanwise wave-number limit, the boundary region solution expands as

$$
\begin{gathered}
\{\bar{u}, \overline{\mathrm{v}}, \bar{w}\}=\left\{\tilde{u}^{(0)}, \widetilde{\mathrm{v}}^{(0)}, \widetilde{w}^{(0)}\right\}+\kappa\left\{\tilde{u}^{(1)}, \widetilde{\mathrm{v}}^{(1)}, \widetilde{w}^{(1)}\right\}+\cdots, \\
\bar{p}=\frac{1}{\kappa} \widetilde{p}^{(0)}+\widetilde{p}^{(1)}+\cdots .
\end{gathered}
$$

Substituting $(6.1)-(6.2)$ into $(5.2)-(5.5)$ shows that the leading-order solution is simply the linear unsteady boundary-layer solution. At 
the next order, the boundary-layer streamwise momentum and continuity equations hold, but the spanwise momentum equation becomes

$$
-i \widetilde{w}^{(1)}+F^{\prime} \frac{\partial \widetilde{w}^{(1)}}{\partial \bar{x}}-\frac{F}{2 \bar{x}} \frac{\partial \widetilde{w}^{(1)}}{\partial \eta}=\widetilde{p}^{(0)}+\frac{1}{2 \bar{x}} \frac{\partial^{2} \widetilde{w}^{(1)}}{\partial \eta^{2}}
$$

with

$$
\widetilde{w}^{(1)} \rightarrow \sqrt{2 \bar{x}}[(i \chi-1)(\eta-\beta)+\tilde{g}(\bar{x})] e^{i \bar{x}}, \eta \rightarrow \infty
$$

and

$$
\tilde{p}^{(0)}=[\sqrt{2 \bar{x}} \widetilde{g}(\bar{x})]^{\prime} e^{i \bar{x}}
$$

where $\chi=\kappa_{2} / \kappa=0(1)$ and $\tilde{g}(\bar{x})$ is determined from the leading order problem as

$$
\widetilde{g}(\bar{x})=\lim _{\eta \rightarrow \infty}\left[\widetilde{\mathbf{v}}^{(0)} e^{-i \bar{x}}+\eta-\beta\right]
$$

The upstream boundary conditions at this order are

$$
\begin{gathered}
\tilde{u}^{(1)} \rightarrow(2 \bar{x})^{3 / 2} Q(\eta) e^{i \bar{x}} \\
\widetilde{v}^{(1)} \rightarrow \sqrt{2 \bar{x}} R(\eta) e^{i \bar{x}} \\
\widetilde{w}^{(1)} \rightarrow \sqrt{2 \bar{x}} S(\eta) e^{i \bar{x}}
\end{gathered}
$$

for $\bar{x} \rightarrow 0$, where $Q, R, S$ are determined by a system of ordinary differential equations determined by substituting (6.7)-(6.9) into the 
governing equations. These are essentially the same as those to be solved in obtaining the second term in the starting conditions for the boundary-region equations solution.

Figures 12 and 13 show results from computations of the leading-order (boundary layer) solution and first-order correction for the streamwise velocity component for $\chi=1$. The results show that $\left|\widetilde{u}^{(1)}\right|$ quickly becomes quite large compared with $\left|\tilde{u}^{(0)}\right|$ so that the expansion (6.1) and (6.2), and hence the boundary-layer approximation, becomes invalid. Equations $(6.3)-(6.5)$ reveal that it is the pressure term $\widetilde{p}^{(0)}$, induced by the leading-order transverse velocity, which causes the rapid breakdown.

The boundary-region (or spanwise ellipticity) effects are seen to come in very strongly, making the boundary-layer approximation valid for only very long spanwise wavelengths or very near the leading edge. It is clear, then, that the analysis of Gulyaev et al. (1989), based on the boundary-layer equations, cannot adequately describe the evolution of Klebanoff modes, whose spanwise length scales are generally found to be of about the order of the boundary-layer thickness, except in a very small region close to the leading edge. 
In the next section, we derive the formulas needed to compute the statistical quantities within the boundary layer using the numerical solutions to the boundary region equations.

\section{Statistical Quantities in the Boundary Layer}

A great advantage of the linear analysis is that the solutions for the individual Fourier components within the boundary layer can be superposed to evaluate the statistical quantities of interest in terms of the upstream turbulence field. The decomposition (4.9)-(4.10) provides the relevant "transfer functions" which relate the fluctuating velocity within the boundary layer to the Fourier coefficients of the upstream turbulence. (Funt, 1973; Goldstein and Durbin, 1980) .

The quantity of most interest is the two-pojnt, time-delayed, streamwise velocity correlation, $R_{11}(\tau, \zeta)$, wrich, as shown by Batchelor and Proudman (1954) and Hunt (1973) can be expressed in terms of the upstream turbulence spectral tensor, $\Phi_{\infty / m}$, as

$$
R_{11}(\varsigma, \tau)=\iint_{-\infty}^{\infty} \int e^{i\left(k_{3} \varsigma-k_{1} \tau\right)} m_{1 \ell} *(x, y, \mathbf{k}) m_{1 m}(x, \nu, \mathbf{k}) \Phi_{\infty \ell m}(\mathbf{k}) d \mathbf{k}
$$

where, in general, 


$$
m_{11}=\bar{u}^{(0)} ; m_{12}=\frac{i k_{1}}{\gamma} \bar{u}^{(0)}-\frac{k_{3}^{2}}{k_{1} \gamma} \bar{u} ; m_{13}=\frac{i k_{3}}{k_{1}} \bar{u}
$$

and the * denotes complex conjugation.

The simplest upstream spectral tensor is probably the one corresponding to isotropic turbulence, namely

$$
\Phi_{\infty i j}=\frac{E(k)}{4 \pi k^{4}}\left(k^{2} \delta_{i j}-k_{i} k_{j}\right)
$$

where $E(k)$ is the three-dimensional spectrum function, $\delta_{i j}$ denotes the Kronecker delta, and $k=\sqrt{k_{1}^{2}+k_{2}^{2}+k_{3}^{2}}$.

Using this, along with (7.2), in (7.1) shows that the two components of the boundary-layer flow contribute separately to the streamwise velocity correlation (Gulyaev et al., 1989) which becomes

$$
R_{11}(\varsigma, \tau)=\frac{1}{4 \pi} \iint_{-\infty}^{\infty} \int \frac{E(k)}{k^{2}} e^{i\left(k_{3} \zeta-k_{1} \tau\right)}\left(\left|\bar{u}^{(0)}\right|^{2}+\frac{k_{3}^{2}}{k_{1}^{2}}|\bar{u}|^{2}\right) d \mathbf{k}
$$

We only consider the contribution to (7.4) of the three-dimensional component $\bar{u}$, which clearly makes up the dominant contribution at low frequency. Changing integration variables using $\bar{x}=k_{1} x$ and using the fact that $\bar{u}\left(-\bar{x}, \eta, i \kappa_{2}, i \kappa\right)=\bar{u}^{*}\left(\bar{x}, \eta, \kappa_{2} \kappa\right)$, which can be shown from the boundary-region equations and boundary conditions, the zero-time-delay correlation becomes 


$$
\begin{aligned}
& R_{11}(\zeta, 0)=\frac{x}{2 \pi} \int_{0}^{\infty} \frac{1}{\bar{x}^{2}} \iint_{-\infty}^{\infty} \frac{E\left(\left(\frac{\bar{x}}{x}\right)^{2}+k_{2}^{2}+k_{3}^{2}\right)}{\left(\frac{\bar{x}}{x}\right)^{2}+k_{2}^{2}+k_{3}^{2}} \\
& e^{i k_{3} \zeta} k_{3}^{2}\left|\bar{u}\left(\bar{x}, \eta, k_{2} \sqrt{\frac{x}{\bar{x} R_{\Lambda}}}, k_{3} \sqrt{\frac{x}{\bar{x} R_{\Lambda}}}\right)\right|^{2} d k_{2} d k_{3} d \bar{x}
\end{aligned}
$$

Introducing the polar coordinates

$k_{2}=\sqrt{k^{2}-\left(\frac{\bar{x}}{x}\right)^{2}} \cos \theta, \quad k_{3}=\sqrt{k^{2}-\left(\frac{\bar{x}}{x}\right)^{2}} \sin \theta$ yields

$$
\begin{aligned}
& R_{11}(\varsigma, 0)=\frac{\delta^{2} R_{\Lambda}}{4 \pi} \int_{0}^{\infty} \int_{\bar{x} / x}^{\infty} \frac{E(k)}{k} \int_{0}^{2 \pi} e^{i\left(\varsigma \sqrt{k^{2}-\left(\frac{\bar{x}}{x}\right)^{2}} \sin \theta\right)} \frac{\left[k^{2}-\left(\frac{\bar{x}}{x}\right)^{2}\right] \sin ^{2} \theta}{\bar{x}^{2}} \\
& \left|\left(\bar{u} \mid \bar{x}, \eta \frac{\sqrt{k^{2}-\left(\frac{\bar{x}}{x}\right)^{2}} \delta \cos \theta}{\sqrt{2 \bar{x}}}, \frac{\left.\sqrt{k^{2}-\left(\frac{\bar{x}}{x}\right)^{2}} \delta \sin \theta\right)}{\sqrt{2 \bar{x}}}\right)\right|^{2} d \theta d k d \bar{x},
\end{aligned}
$$

where

$$
\delta=\sqrt{\frac{2 x}{R_{\wedge}}}
$$

is the local boundary-layer thickness. 
For isotropic turbulence, the three-dimensional spectrum function, $E(k)$ is related to the one-dimensional spectrum function, $E_{1}(k)$, by

$$
E(k)=k^{3} \frac{d}{d k} \frac{1}{k} \frac{d E_{1}}{d k}
$$

Using this expression in (7.6) and integrating twice by parts yields

$$
\begin{aligned}
& R_{11}(\zeta, 0)=\frac{\delta^{2} R_{\Lambda}}{4 \pi} \int_{0}^{\infty} \frac{1}{\bar{x}^{2}}\left\{\frac{1}{2}\left(\frac{\bar{x}}{x}\right)^{2} E_{1}(\bar{x} / x)|\bar{u}(\bar{x}, \eta, 0,0)|^{2}+\int_{\bar{x} / x}^{\infty} E_{1}(k) \frac{d}{d k} \frac{1}{k} \frac{d}{d k} k^{2}\left(k^{2}-(\bar{x} / x)^{2}\right)\right. \\
& \int_{0}^{2 \pi} e^{i \zeta \sqrt{k^{2}-(\bar{x} / x)^{2}} \sin ^{2} \theta} \sin ^{2} \theta \mid\left(\bar{u} \mid \bar{x}, \eta, \frac{\sqrt{k^{2}-\left(\frac{\bar{x}}{x}\right)^{2}} \delta \cos \theta}{\sqrt{2 \bar{x}}}, \frac{\left.\sqrt{k^{2}-\left(\frac{\bar{x}}{x}\right)^{2}} \delta \sin \theta\right)\left.\right|^{2}}{\sqrt{2 \bar{x}}} d \theta d k\right\} d \bar{x} .
\end{aligned}
$$

Our interest here is in the downstream (low frequency) limit $\frac{\bar{x}}{x} \rightarrow 0$ of the urms in the boundary layer

$$
R_{11}(0,0)=\frac{\delta^{2} R_{\Lambda}}{4 \pi} \int_{0}^{\infty} k E_{1}(k) K(\eta, k ; \delta) d k
$$

where

$$
K(\eta, k, \delta)=\frac{1}{k} \frac{\partial}{\partial k} \frac{1}{k} \frac{\partial}{\partial k} k^{4} \int_{0}^{\infty} \int_{0}^{2 \pi} \frac{1}{\bar{x}^{2}}\left|\bar{u}\left(\bar{x}, \eta, \frac{k \delta \cos \theta}{\sqrt{2 \bar{x}}}, \frac{k \delta \sin \theta}{\sqrt{2 \bar{x}}}\right)\right|^{2} \sin ^{2} \theta d \theta d \bar{x}
$$


Since it is very difficult to generate truly isotropic turbulence in a laboratory experiment, Batchelor (1946, 1953) and Chandrasekhar (1950) proposed a less restrictive, axisymmetric turbulence model. In Appendix $C$, we show that the corresponding spectral tensor can be written as

$$
\begin{aligned}
& \Phi_{\infty i j}=\frac{1}{k_{\perp}^{2}}\left(\Phi_{t}-\frac{2 k_{1}^{2}}{k_{\perp}^{2}} \Phi_{1}\right)\left(k_{\perp}^{2} \delta_{i j}^{\perp}-k_{\perp j} k_{\perp j}\right) \\
& +\frac{1}{k_{\perp}^{2}} \Phi_{1}\left(k_{1}^{2} \delta_{i j}^{\perp}-k_{1} k_{\perp i} \delta_{j 1}-k_{1} k_{\perp j} \delta_{i 1}+k_{\perp}^{2} \delta_{i 1} \delta_{j 1}\right),
\end{aligned}
$$

where

$$
k_{\perp i} \equiv k_{i}-\delta_{i 1} k_{1}
$$

are the cross-stream, wave-number components,

$$
\delta_{i j}^{\perp} \equiv \delta_{i j}-\delta_{i 1} \delta_{j 1}
$$

is the cross-stream Kronecker delta, $k_{\perp}=\sqrt{k_{2}^{2}+k_{3}^{2}}$, and the scalars $\Phi_{1}\left(k_{1}, k_{\perp}\right)$ and $\Phi_{1}\left(k_{1}, k_{\perp}\right)$ are related to the longitudinal and lateral onedimensional spectrum functions $E_{1}\left(k_{1}\right)$ and $E_{t}\left(k_{1}\right)$, respectively, by

$$
E_{1}\left(k_{1}\right)=2 \pi \int_{0}^{\infty} \Phi_{1} k_{\perp} d k_{\perp}
$$

and 


$$
E_{t}\left(k_{1}\right)=2 \pi \int_{0}^{\infty} \Phi_{t} k_{\perp} d k_{\perp}
$$

In this case, there is a contribution to the streamwise velocity correlation from cross products of the $\bar{u}$ and $\bar{u}^{(0)}$ components. But again, we consider only the dominant $\bar{u}$ contribution. Then using (7.12) in (7.1), with (7.2), changing variables of integration, and taking the low-frequency limit, as in the isotropic case, shows that the mean square of the streamwise velocity fluctuation can be written as

$$
R_{11}(0,0)=\delta^{2} R_{\Lambda} \int_{0}^{\infty} k_{\perp}^{3} \Phi_{t}\left(0, k_{\perp}\right) K_{0}\left(\eta, k_{\perp} \delta\right) d k_{\perp}
$$

where

$$
K_{0}\left(\eta, k_{\perp} \delta\right)=\int_{0}^{\infty} \int_{0}^{2 \pi} \frac{\sin ^{2} \theta}{\overline{\mathrm{x}}^{2}}\left|\bar{u}\left(\bar{x}, \eta, \frac{k_{\perp} \delta \sin \theta}{\sqrt{2 \bar{x}}}, \frac{k_{\perp} \delta \cos \theta}{\sqrt{2 \bar{x}}}\right)\right|^{2} d \theta d \bar{x}
$$

These results show that only the transverse spectral function, $\Phi_{t}$, of the upstream turbulence is of direct relevance to the generation and growth of disturbances in the boundary layer, as opposed to the longitudinal spectrum function which is most often documented in the experiments. The former function can be determined from measurements of the transverse correlation function

$$
R_{\infty \perp}\left(\xi-\tau, r_{\perp}\right)=\overline{u_{\perp}(x-t, y, z) u_{\perp}\left(x^{\prime}-t^{\prime}, y^{\prime}, z^{\prime}\right)}
$$


where $\xi=x^{\prime}-x, \quad \tau=t^{\prime}-t$, and $\quad r_{\perp}=\sqrt{\left(y^{\prime}-y\right)^{2}+\left(z^{\prime}-z\right)^{2}}$,

by taking the Hankel transformation of its longitudinal Fourier transform

$$
\Phi_{1}\left(k_{1}, k_{\perp}\right)=2 \int_{0}^{\infty} r_{\perp} \int_{-\infty}^{\infty} R_{\infty \perp}\left(\xi-\tau, r_{\perp}\right) e^{i(\xi-\tau) k_{1}} d(\xi-\tau) J_{0}\left(r_{\perp} k_{\perp}\right) d r_{\perp}
$$

As far as we know, no such measurements have been made, so the form of this function is still unknown and may differ considerably from one experiment to another. Moreover, the present results show that only the low-frequency portion of $\Phi_{t}$ determines the velocity fluctuations in the boundary layer, which is the part of the spectrum that is likely to be highly anisotropic (Batchelor, 1956, p. 91)--retaining a history of the upstream screens or grid which produced it. The low-frequency spectral components should therefore possess a relatively high degree of periodicity in the transverse wave number. The extreme limit of this is described by

$$
\Phi_{t}\left(0, k_{\perp}\right)=\frac{2 u_{\infty}^{2}}{\pi^{2}} \delta\left(k_{\perp}^{2}-k_{t}^{2}\right)
$$

where $\delta$ denotes the Dirac delta function, $k_{t}$ characterizes the periodicity of the low-frequency turbulence, and the constant factor was chosen so that (7.16) satisfies the isotropic relation $E_{t}(0)=2 u_{\infty}^{2} / \pi$, in order to be consistent with the measurements shown in Figure 4. of Westin et al. (1994). 
Using (7.21) in (7.17) leads to the simple expression

$$
\frac{R_{11}(0,0)}{u_{\infty}^{2} R_{\Lambda}}=\left[\frac{\left(k_{t} \delta\right)}{\pi}\right]^{2} K_{0}\left(k_{t} \delta\right)
$$

The numerical results are presented and compared with experimental measurements in the next section.

\section{Numerical Results and Comparisons with Experimental Data}

Numerical computations of the individual Fourier component solutions of the linearized unsteady boundary-region equations were carried out over a broad range of scaled wave numbers, $\boldsymbol{k}_{2}$ and $\boldsymbol{k}$, in order to calculate the mean square of the streamwise velocity fluctuations in the boundary layer. The results for isotropic free-stream turbulence are presented first. A number of different functional forms have been proposed for the corresponding one-dimensional spectrum function $E_{1}$. They are usually obtained by fitting of experimental data or from assumptions about the correlation function behavior. Figure 14 shows some numerical results for the normalized root mean square (rms) of the streamwise velocity fluctuations, $u^{\prime} / u_{\infty} \sqrt{R_{\Lambda}}$, at fixed $\eta=1.64$ (which closely corresponds to the peak location of the streamwise 
velocity fluctuations). They are based on the one-dimensional spectrum proposed by Gulyaev et al (1989)

$$
E_{1}(k)=\frac{2 u_{\infty}^{2}}{\pi} \frac{e^{-6.7 k / r_{1}^{3 / 4}}}{1+b(2 k)^{5 / 3}}
$$

where $b=1.35 /\left(1+35 /\left(2 r_{t}\right)^{3 / 4}\right)$, with our normalization. For isotropic turbulence, the normalized rms streamwise velocity fluctuation depends on the single parameter $r_{t}$, which enters only through the one-dimensional spectrum.

Notice that the normalized rms velocity fluctuation initially increases linearly with $\delta$, which corresponds to the result obtained by using solutions of the linearized boundary-ayer equations in place of the boundary-region solutions. However, the spanwise ellipticity effects quickly cause the growth to decrease below the linear boundary-layer results, with the rms velocity fluctuations reaching a peak and then decreasing with further increase of $\delta$. This is because, even for small $\delta$, the boundary-region solutions selected by the integration do not correspond to small values of $k$ and $\kappa_{2}$. All the curves reach approximately the same peak level, but those at larger turbulent Reynolds numbers have greater initial slopes and peak at smaller values of $\delta$. These results show thet the magnitude of the streamwise velocity fluctuations in the bouridary layer can reach three or four times the free-stream level at $R_{\Lambda}$ values on the order of a few thousand, which is consistent with the experimentally observed Klebanoff mode amplitudes found in the more recent experiments. 
The most well-documented of these experiments are those of Roach and Brierley (1992) and Westin et al. (1994). The investigators attempted to make the incident turbulent flow as isotropic as possible in both of these studies. However, it is very difficult to control the lowfrequency component of the spectrum that actually enters the boundary layer to produce the Klebanoff modes. Watmuff (1997) was able to reduce the peak rms velocity in the boundary layer by as much as 50 percent by reordering the screens upstream of his test section according to the quality of their spanwise uniformity. This suggests that even relatively minor changes in the low-frequency spectral characteristics of the free-stream turbulence can produce large differences in the rms velocity in the boundary layer. The inevitable deviations from pure isotropy that occur at low frequencies in any experiment may therefore have an important effect on the velocity fluctuations in the boundary layer.

Westin et al. (1994) conducted experiments at free-stream turbulence levels of 1.35 percent and 1.5 percent. They estimated the transverse integral scale of their free-stream turbulence to be between 7 and $10 \mathrm{~mm}$, based on extrapolations of their measured spectra. In the following comparisons, we use a value of $8 \mathrm{~mm}$, which corresponds to an $R_{\Lambda}$ value of 2120 for their 1.35 percent run (with $U_{\infty}=4 \mathrm{~m} / \mathrm{s}$ ) and 4240 for the 1.5 percent case $\left(U_{\infty}=8 \mathrm{~m} / \mathrm{s}\right)$. The corresponding values of $r_{t}$ are 29 and 64 for the 1.35 percent and 1.5 percent cases, respectively. 
The measurement points were in the range of $156 \mathrm{~mm}$ to $460 \mathrm{~mm}$ downstream of the leading edge in the 1.35 percent case and between $102 \mathrm{~mm}$ and $1000 \mathrm{~mm}$ in the 1.5 percent case. The corresponding scaled streamwise variable $\varepsilon x^{*} / \Lambda$ varied between 0.3 and 0.8 in the 1.35 percent case and 0.2 and 2.0 in the 1.5 percent case. This suggests that the linear theory may still be applicable to the data points closest to the leading edge even though the turbulent Reynolds number is not particularly small in these experiments. However, the data points lying further downstream probably extend into the nonlinear regime--in fact, beyond the point where a singularity was found to develop in the steady boundary-layer solution (Goldstein et al., 1992, and Goldstein and Leib, 1993).

Figure 15 shows comparisons of the linear theory predictions with the data of westin et al. (1994). The dashed curves, which correspond to isotropic free-stream turbulence, fairly accurately predict the initial growth rate in the experiments. However, the strong spanwise ellipticity effects in the boundary-region equations quickly cause the growth to diminish, and the curves reach a more or less constant level. While this level is below that of the experimental data, it is of about the right order of magnitude. The agreement with the data is better at smaller $\delta$ and, overall, at smalles turbulent Reynolds number, consistent with expectations for the linear theory. 
The solid curves in Figure 15 were computed from (7.20) with $k_{t}$, and therefore the periodicity of the low-frequency turbulence, chosen to achieve the maximum level of $u$ rms. The results show greatly improved agreement with the data as compared with the calculation for isotropic free-stream turbulence. The deviation from the data is again maximum at larger turbulent Reynolds numbers and at larger downstream distances from the leading edge. Of course, the turbulent energy is distributed over a finite wave-number band in the actual experiments, and calculations based on an experimentally measured form of $\Phi_{t}$, if it were available, would probably lie in between the solid and dashed curves in Figure 15.

Roach and Brierley (1992) carried out experiments over a fairly broad range of free-stream turbulence levels with a variety of turbulencegenerating grids. We compare our linear theory with data from four of their runs--the others having either no organizing grid or extremely high turbulence levels. Roach (1987) presents detailed measurements of the intensities and scales of the turbulence generated by the grids used in the Roach and Brierley (1992) experiments and suggests empirical formulas for their description. Using these relations, we estimate the turbulence intensity at the leading edge to be about 0.8 percent for their SMR grid and 3 percent for their PR grid. The corresponding transverse integral scales are approximately $2.4 \mathrm{~mm}$ and $6.0 \mathrm{~mm}$ for the SM and PR grids, respectively. Runs were made with two different free-stream speeds for each turbulence grid. At the lower speed of $14.9 \mathrm{~m} / \mathrm{s}$ with the SMR grid (the SMRLO case) $\mathrm{R}_{\Lambda}$ is 
approximately equal to 2400 , and $r_{t}$ is apprcximately equal to 19 . For the second run with the SMR grid, called the SMRHI case, the freestream velocity was $19.8 \mathrm{~m} / \mathrm{s}, R_{\Lambda} \approx 3200$, and $r_{t} \approx 26$. The PR grid was run at $3 \mathrm{~m} / \mathrm{s}$ (PRLO), corresponding to $R_{\Lambda} \approx 1200, r_{t} \approx 36$ and at $5.3 \mathrm{~m} / \mathrm{s}$, PRHI, with $R_{\Lambda} \approx 2100$ and $r_{t} \approx 63$. The measurement locations recovered from these estimates ranged roughly between $85 \mathrm{~mm}$ and $1.1 \mathrm{~m}$ from the leading edge for the SMR grid, corresponding to scaled distances $\varepsilon x^{*} / \Lambda$ from 0.3 to 4.0 . With the PR grid, the streamwise locations ranged from $41 \mathrm{~mm}$ to near the end of the plate $(1.7 \mathrm{~m})$ so that $\varepsilon x^{*} / \Lambda$ ranged from 0.2 to 8.5. This data then, like the Westin et al. (1994) data, encompasses a region where we would expect the linear theory to apply, but also extends well into the nonlinear regime.

Figure 16 shows the comparisons of the linear theory with the Roach and Brierley (1992) data. The results are similar to the westin et al. (1994) comparison. The isotropic curves (dashed) begin with about the right growth rate, and the greatest deviation is at large $\delta$ and $r_{t}$. The solid curves are again computed from the strongly anisotropic model and show improved agreement with the data.

These results suggest that low-frequency ansotropy, which is certainly present in all experiments to some extent, could potentially play an important role in the generation of the large amplitude streamwise velocity fluctuations associated with the Klebanoff modes. 
There are, of course, additional effects which are present, to varying degrees in these experiments, some of which are discussed in the next section. We note here, however, that westin et al. (1994) report observing turbulent spots near their last measurement station at the higher turbulence level. These must certainly be preceded by nonlinear effects in the region upstream, and we would similarly expect spots to have appeared in the PRHI data of Roach and Brierley $(1992)\left(r_{t}=63\right)$.

\section{Discussion}

We have carried out a systematic linear analysis of the effects of vortical free-stream disturbances on a laminar flat plate boundary layer. The upstream distortion was decomposed into its various Fourier components. The analysis describes the resulting downstream evolution of the flow, first through an unsteady boundary-layer region and then into a region where spanwise ellipticity effects are important. The flow in the latter region is governed by the unsteady boundary-region equations, which were solved numerically subject to upstream and farfield boundary conditions derived from strict asymptotic matching of the solutions in the various regions shown in Figure 1. The spanwise ellipticity effects are surprisingly strong and very quickly influence the growth of the disturbances. The linearized approximation allows us to superpose the individual Fourier component solutions to the unsteady boundary-region equations and thereby calculate the rms 
streamwise velocity fluctuations in the boundary layer due to a broadband external turbulent flow. Results were obtained for both pure isotropic turbulence and for axisymmetric turbulence with strong low-frequency anisotropy.

Comparison of the theoretical calculations with recent experimental data shows that the disturbances produced by the linear mechanism described above closely resemble the behavior actually observed for Klebanoff modes. The theoretical results for isotropic turbulence predict the initial linear growth with boundary-layer thickness but, due to the strong pressure coupling effects in the boundary-region equations, the amplitudes do not reach the levels found in the experiments. Calculations using a strongly anisotropic model for the free-stream turbulence suggests that low-frequency anisotropic effects could be a significant factor in explaining the discrepancy.

Bertolotti (1997) carried out an ad hoc ana...ysis of the problem considered in this paper by using the parabolic stability equations. Results from calculations with a single, steady, free-stream mode were compared with the data of Westin et al. (1994), and agreement was obtained by selecting the modal amplitude to produce the best results. Bertolotti (1997) also made comparisons with recent, unpublished, data of Kendall. Of particular interest is the comparison with filtered rms data for various frequency bands. The computations qualitatively predict the large amplification of the low-Erequency components that 
was observed in the experiment, but generally underpredict the actual amplitudes with the agreement being worse at large downstream distances and frequencies. The calculations were again made with a single (spanwise and transverse) free-stream mode of pre-selected amplitude.

Our analysis differs from that of Bertolotti (1997) in a number of important aspects. First, we have derived our governing equations and boundary conditions in a rational way from the Navier-stokes equations. Second, we have made use of the major attraction of the linear theory, namely, its superposition feature to combine the individual Fourier component solutions to obtain results corresponding to the actual broadband turbulent flow. Moreover, our comparisons with experimental data use the reported free-stream turbulence levels as the overall input into the linear theory.

As already mentioned, the analysis is restricted to what appears to be the dominant generation and amplification mechanism of low-frequency disturbances in the boundary layer, viz. the linear amplification of crossflow-driven disturbances in the boundary layer on an infinitely thin flat plate. There are numerous other effects present in the experiments which might potentially contribute to the amplification of the disturbances. 
our calculations of $u$ rms only include the $\bar{u}$ contribution. The $\bar{u}^{(0)}$ component makes an independent, and much smaller, contribution to the rms velocity fluctuation (Gulyaev et al. 1989) for isotropic free-

stream turbulence. However, in the axisymmetric turbulence approximation there are cross-product contributions to the rms velocity due to the interaction between $\bar{u}$ and $\bar{u}^{(0)}$ components which would probably yield a larger contribution than that due to the selfinteraction of $\bar{u}^{(0)}$.

Kendall (1991) and Watmuff (1997) found that changing the leading-edge bluntness of their plates had very little effect on the amplitude or spanwise spacing of the disturbances in the boundary layer. However, stretching of vortex lines initially normal to the plate by a relatively blunt leading edge produces a streamwise vorticity (crossflow) which is then imposed on the boundary-layer flow (Goldstein et al., 1992; Goldstein and Wundrow, 1997). This mechanism leads to augmentation of the disturbance amplification relative to that of an infinitely thin plate. It is worth noting that the leading-edge bluntness effects were not investigated in the experiments of Roach and Brierley (1992) and Westin et al. (1994).

As already noted, the governing equations show that nonlinear effects become important when $\varepsilon x=0(1)$. Results from numerical calculations of the steady three-dimensional, boundary-layer and boundary-region equations in Goldstein et al. (1992), Goldstein and Leib (1993), and 
Goldstein and Wundrow (1997) show that the boundary layer responds more or less linearly for some distance, up to $\mathrm{ex}$ of around 0.6 and that nonlinear effects, when they become significant, enter very rapidly. In the experiments of Roach and Brierley (1992) and Westin et al (1994), Ex ranges from about 0.2 at the initial measurement station to about 3 at the most downstream measurement point where comparisons with the linear theory are presented. We therefore expect the linear theory to be capable of describing at least the initial stage of Klebanoff-mode evolution. The appearance of turbulent spots in the data of Westin et al. (1994), however, clearly shows that nonlinearity eventually becomes important.

Additional theoretical and experimental work is required before a full understanding of the Klebanoff-mode generation and growth mechanisms is in hand. On the theoretical side, the additional effects discussed above could each be analyzed separately, and then superposed when linear theory can be used. However, it is probably more important to account for nonlinear effects. A major difficulty with this is that it is no longer possible to superpose free-stream modes in order to simulate a homogeneous external turbulent flow. A full simulation of the problem, including the free-stream turbulence, is impractical with current computational capabilities, so that some type of modeling of the free-stream turbulence would be needed. Unfortunately, this has not been adequately developed, but perhaps the linear theory, together with experimental data, could be of some use to guide in the selection of an appropriate model. 
On the experimental side, our analysis suggests that important information could be obtained from detailed measurements of the lowfrequency portion of the free-stream turbulence spectrum and, in particular, the relative degree of anisotropy therein. In addition, measurements of the transverse correlation Eunction $R_{v t}$ of the freestream turbulence would provide the input needed to compute the boundary-layer $u$ rms in our axisymmetric model. This would allow a more definitive test of the present linear theory and might suggest further lines of research to develop a fuller understanding of this phenomena.

The exact role played by the Klebanoff mode in the laminar-turbulent transition process remains unclear. They can reach very large amplitudes in the boundary layer before transition occurs, whereas TS waves provoke transition at much lower leve...s. Experiments by Boiko et al. (1994) show that sufficiently high levels of free-stream turbulence can produce significant transfer of energy between frequencies within the unstable bands for TS waves. It is therefore possible that the Klebanoff modes primarily influence transition by modifying the base flow, which, among other things, causes a broadening of the frequency band over which the TS waves can grow. A stability analysis of such a base flow, i.e., the Blasius profile with Klebanoff modes superposed, could shed additional light on this issue. Wundrow and Goldstein (1994), Wundrow (1996:, and Goldstein and Wundrow (1995) have already made some progress along these lines. 
Another possibility is that large-amplitude Klebanoff modes may generate turbulent spots directly. Finally, it is worth noting that the Klebanoff modes exhibit a marked similarity to the sublayer streaks in the turbulent boundary layer: a connection that definitely merits further investigation.

\section{Appendix A: Edge-Layer Solution for Asymptotic LUBI Equations}

Equation (4.22) suggests that the appropriate transverse coordinate in the edge layer is given by

$$
\bar{\eta} \equiv \eta-\beta=\eta_{0}+\delta_{0} \hat{\eta}
$$

where $\eta_{0}$ is determined by

$$
2 \bar{x} A=\eta_{0}^{3} e^{\eta_{0}^{2} / 2}
$$

and $\delta_{0}(\bar{x}) \delta \Lambda$ is the edge-layer thickness. Then the mean velocity will exhibit an order-one variation across the edge layer if we put

$$
\delta_{0} \eta_{0}=1
$$

Substituting these into the unsteady boundary-layer equations, and (4.10) show that

$$
\{\bar{u}, \overline{\mathrm{v}}, \bar{w}\}=\left\{\bar{u}_{e}, \delta_{0} \overline{\mathrm{v}}_{e}, \bar{w}_{e}\right\} e^{i \bar{x}}
$$

are determined by 


$$
\begin{gathered}
\left(i \bar{u}_{e}-\bar{v}_{e}\right) e^{-\hat{\eta}}+\frac{\partial \bar{u}_{e}}{\partial \hat{\eta}}+\frac{\partial^{2} \bar{u}_{e}}{\partial \hat{\eta}^{2}}=0 \\
i \bar{w}_{e} e^{-\hat{\eta}^{2}}+\frac{\partial \bar{w}_{e}}{\partial \hat{\eta}}+\frac{\partial^{2} \bar{w}_{e}}{\partial \hat{\eta}^{2}}=0 \\
i \bar{u}_{e}+\frac{\partial \bar{v}_{e}}{\partial \hat{\eta}}+\bar{w}_{e}=0
\end{gathered}
$$

subject to the boundary condition that

$$
\bar{u}_{e} \rightarrow 0 \quad \bar{w}_{e} \rightarrow 1 \quad \text { as } \quad \hat{\eta} \rightarrow \infty
$$

and that $\bar{w}_{e} e^{\bar{x}}$ match onto (4.18) as $\hat{\eta} \rightarrow-\infty$ with a similar condition for $\bar{u}_{e}$. As suggested by Gulyaev et al. (1989), (A-7) can easily be solved in terms of Hankel functions to obtain

$$
\bar{w}_{e}=-\frac{\pi}{\sqrt{i}} e^{-\tilde{\eta}^{\prime} / 2} H_{1}^{(1)}\left(2 \sqrt{i} e^{-\hat{\eta} / 2}\right),
$$

which clearly satisfies the outer-edge boundary condition (A-8), while the large argument expansion for $\mathrm{H}_{1}{ }^{(1)}$ shows that

$$
\begin{aligned}
& \bar{w}_{e} \rightarrow \sqrt{\pi} e^{-i \pi / 8} e^{-\hat{\eta} / 4} e^{-2 \sqrt{i} e^{-\hat{\eta} / 2}} \\
& \text { as } \hat{\eta} \rightarrow-\infty
\end{aligned}
$$

which, in view of $(A-1)-(A-4)$ and $(4.18)-(4.20)$, will clearly match onto (4.18) if we take 


$$
c_{0}=\sqrt{\frac{\pi}{i}} A^{\frac{1}{2}}(2 \bar{x})^{\frac{1}{4}} e^{-H_{\infty}+i \pi / 8}
$$

where $H_{\infty} \equiv \lim _{\bar{\eta} \rightarrow \infty}\left[H+\frac{1}{4} \bar{\eta}^{2}+\ln \bar{\eta}\right]$.

\section{Appendix B: Axisymmetric Turbulence}

The general form of the energy spectrum tensor in axisymmetric turbulence is (Batchelor, 1946, 1953; Chandrasekhar, 1950)

$$
\Phi_{i j}=A k_{i} k_{j}+B \delta_{i 1} \delta_{j 1}+C \delta_{i j}+D k_{i} \delta_{j 1}+E k_{j} \delta_{i 1}
$$

where $\mathrm{A}, \mathrm{B}, \mathrm{C}, \mathrm{D}$, and $\mathrm{E}$ are scalar functions of $\mathrm{k}_{1}$ and $k^{2}=k_{1}^{2}+k_{2}^{2}+k_{3}^{2}$.

The number of arbitrary functions can be reduced by using the conditions of incompressibility to show that the spectrum tensor can be expressed in terms of two scalar functions

$$
\Phi_{i j}=\frac{\widetilde{A}}{4 \pi k^{4}}\left(k^{2} \delta_{i j}-k_{i} k_{j}\right)+\frac{\widetilde{D}}{4 \pi k^{4}}\left(k_{1}^{2} \delta_{i j}+k^{2} \delta_{i 1} \delta_{j 1}-k_{1} k_{i} \delta_{j 1}-k_{1} k_{j} \delta_{i 1}\right)
$$

Defining,

$$
\Phi_{1}\left(k_{1}, k_{\perp}\right)=\Phi_{11}
$$

and 


$$
\Phi_{t}\left(k_{1}, k_{\perp}\right)=\Phi_{22}+\Phi_{33}
$$

(B.2) can be written as

$$
\Phi_{i j}=\frac{1}{k_{\perp}^{2}}\left(\Phi_{1}-\frac{2 k_{1}^{2}}{k_{\perp}^{2}} \Phi_{1}\right)\left(k_{\perp}^{2} \delta_{i j}^{\perp}-k_{\perp j} k_{\perp j}\right)+\frac{1}{k_{\perp}^{2}} \Phi_{1}\left(k_{1}^{2} \delta_{i j}^{\perp}-k_{1} k_{\perp j} \delta_{j 1}-k_{1} k_{\perp j} \delta_{i 1}+k_{\perp}^{2} \delta_{i 1} \delta_{j 1}\right)
$$

where

$$
k_{\perp i}=k_{i}-\delta_{i 1} k_{1}
$$

$k_{\perp}^{2} \equiv k_{2}^{2}+k_{3}^{2}$, and

$$
\delta_{i j}^{\perp}=\delta_{i j}-\delta_{i 1} \delta_{j 1}
$$

The functions $\Phi_{1}$ and $\Phi_{t}$ are related to the longitudinal and lateral one-dimensional spectra, $E_{1}\left(k_{1}\right)$ and $E_{t}\left(k_{1}\right)$, respectively by

$$
E_{1}\left(k_{1}\right)=2 \pi \int_{0}^{\infty} \Phi_{1}\left(k_{1}, k_{\perp}\right) k_{\perp} d k_{\perp}
$$

and

$$
E_{t}\left(k_{1}\right)=2 \pi \int_{0}^{\infty} \Phi_{t}\left(k_{1}, k_{\perp}\right) k_{\perp} d k_{\perp}
$$

\section{References}

Arnal, D. and Juillen, J. C. (June 1978). Contribution experimentalea l'etude de la receptivite d'une couche limite laminare, a la turbulence de l'ecoulement general. ONERA Rapport Technique No. $1 / 5018$ AYD. 
Batchelor, G. K. (1946). The theory of axisymmetric turbulence. Proc. Roy. SoC. London A, 186, 480.

Batchelor, G. K. (1953). The Theory of Homogeneous Turbulence. Cambridge University Press.

Batchelor, G. K. and Proudman, I. (1954). The effect of rapid distortion of a fluid in turbulent motion. Quart. J. Mech. Appl. Math. 7, 83 .

Bertolotti, F. P. (1997). Response of the Blasius boundary layer to free-stream vorticity. Phys. Fluids 9 (8), 2286-2299.

Boiko, A. V., Westin, K. J. A., Klingmann, B. G. B., Koslov, V. V. and Alfredsson, P. H. (1994). Experiments in a boundary layer subjected to free stream turbulence, Part 2. The role of TS waves in the transition process. J. Fluid Mech. 281, 219-245.

Bradshaw, P. (1965). The effect of wind-tunnel screens on nominally two-dimensional boundary layers. J. Fluid Mech. 22, part 4, 679-687.

Chandrasekhar, S. (1950). The theory of axisymmetric turbulence. Phil. Trans. Roy. Soc. A, 242, 557.

Choudhari, M. (1996). Boundary layer receptivity to three-dimensional unsteady vortical disturbances in free stream. AIAA paper 96-0181 (and High Technology, Inc. report HTC-95051).

Crow, S. C. (1966) The spanwise perturbation of two-dimensional boundary layers. J. Fluid Mech. 24, 153-164.

Darwin, C. (1953). A note on hydrodynamics. Proc. Camb. Phil. Soc. 49, $342-354$.

Davis, R. T. and Rubin, S. G. (1980). Non-Navier stokes viscous flow computations. Comput. Fluids 8, 101.

Dryden, H. L. (1936). Air flow in the boundary layer near a plate. NACA report no. 562 .

Goldstein, M. E. (1978). Unsteady vortical and entropic distortions of potential flows round arbitrary obstacles. J. Fluid Mech. 89, 433-468.

Goldstein, M. E. (1983). The evolution of Tollmien-Schlichting waves near a leading edge. J. Fluid Mech. 127, 59-81.

Goldstein, M. E. and Durbin, P. A. (1980). The effect of finite turbulence spatial scale on the amplification of turbulence by a contracting stream. J. Fluid Mech. 98, part 3, 473-508.

Goldstein, M. E., Leib, S. J. and Cowley, S. J. (1992). Distortion of a flat plate boundary layer by free stream vorticity normal to the plate. J. Fluid Mech. 237, 231-260. 
Goldstein, M. E. and Leib, S. J. (1993). A note on the distortion of a flat-plate boundary layer by free steam vorticity normal to the plate. J. Fluid Mech. 248, 531-541.

Goldstein, M. E. and Wundrow, D. W. (1995) Interaction of oblique instability waves with weak streamwise vortices. J. Fluid Mech., 284, $377-407$.

Goldstein, M. E. and Wundrow, D. W. (1997). On the environmental realizability of algebraically growing disturbances and their relation to Klebanoff modes. To appear in Theoretical and Computational Fluid Dynamics.

Gulyaev, A. N., Kozlov, V. E., Kuznetson, V. R., Mineev, B. I. and Sekundov, A. N. (1989). Interaction of a laninar boundary layer with external turbulence. Izv. Akad. Nauk SSSR Mekh. Zhid. Gaza 6, $700-710$.

Hunt, J. C. R. (1973). A theory of turbulent flow round twodimensional bluff bodies. J. Fluid Mech. 61, Part 4, 625-706.

Kemp, N. (1951) The laminar three-dimensional boundary layer and a study of the flow past a side edge. M.Ac.S. Thesis, Cornell University.

Kendall, J. M. (July 1985). Experimental study of disturbances produced in a pre-transitional laminar boundary layer by weak free stream turbulence. AIAA paper 85-1695.

Kendall, J. M. (1990). Boundary layer receptivity to free stream turbulence. AIAA paper 90-1504.

Kendall. J. M. (1991). Studies on Laminar boundary layer receptivity to freestream turbulence near a leading edge. In Boundary Layer stability and Transition to Turbulence. ASIME FED - Vol. 114.

Klebanoff, P. S. (1971). Effect of free-stream turbulence on a laminar boundary layer. Bulletin Am. Phys. Soc. 16

Leventhal, L. and Reshotko, E. (1981). Preliminary experimental study of disturbances in a laminar boundary layer due to distributed surface roughness. AIAA paper 81-1224.

Lighthill, M. J. (1956). Drift. J. Fluid Mech. 1, 31-53.

Roach, P. E. (1987). The generation of near y isotropic turbulence by means of grids. Int. J. of Heat and Fluid Plow 8 , \#2. 
Roach, P. E. and Brierley, D. H. (1992) The influence of a turbulent free stream on zero pressure gradient transitional boundary layer development. Part I: Test cases T3A and T3B. In Numerical Simulation of Unsteady Flows and Transition to Turbulence. Cambridge University Press.

Suder, K. L., O'Brien, J. E. and Reshotko, E. (1988). Experimental study of bypass transition in a boundary layer. NASA Tech Mem. 100913 .

Taylor, G. I. (1939). Some recent developments in the study of turbulence. Proceedings of the Fifth International Congress for Applied Mechanics. Cambridge, Mass.

Van Dyke, M. D. (1975). Perturbation Methods in Fluid Mechanics. Parabolic Press.

Westin, K. J. A., Boiko, A. V., Klingmann, B. G. B., Kozlov, V. V. and Alfredsson, P. H. (1994). Experiments in a boundary layer subjected to free stream turbulence. Part 1. Boundary layer structure and receptivity. J. Fluid Mech. 281, 193-218.

Watmuff, J. H. (1997). Detrimental effects of almost immeasurably small free-stream nonuniformities generated by wind tunnel screens. AIAA 97-0228.

Wundrow, D. W. (1996). Linear instability of a uni-directional transversely sheared mean flow. NASA CR 198535.

Wundrow, D. W. and Goldstein, M. E. (1994). Nonlinear instability of a uni-directional transversely sheared mean flow. NASA TM 106779. 


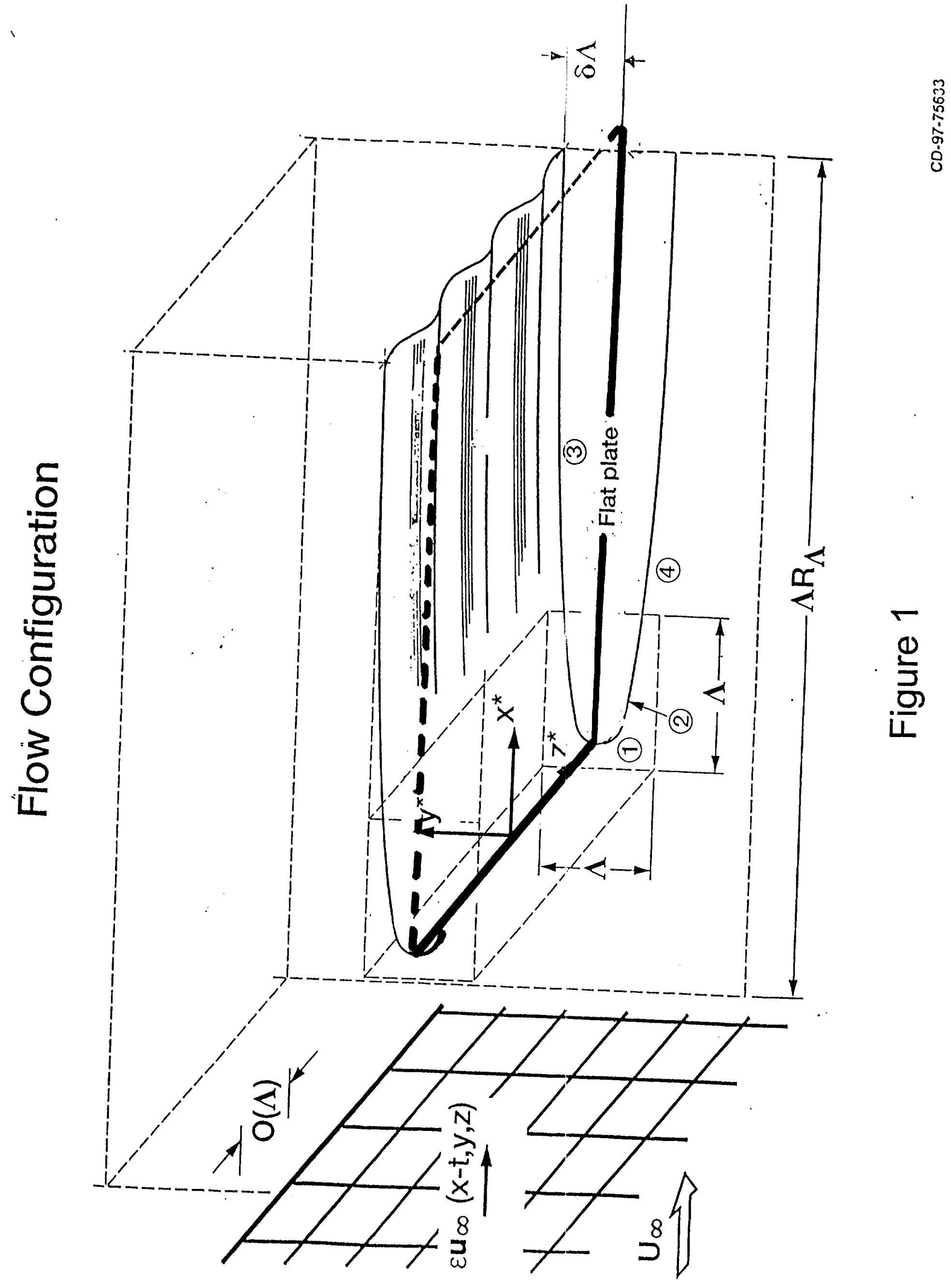



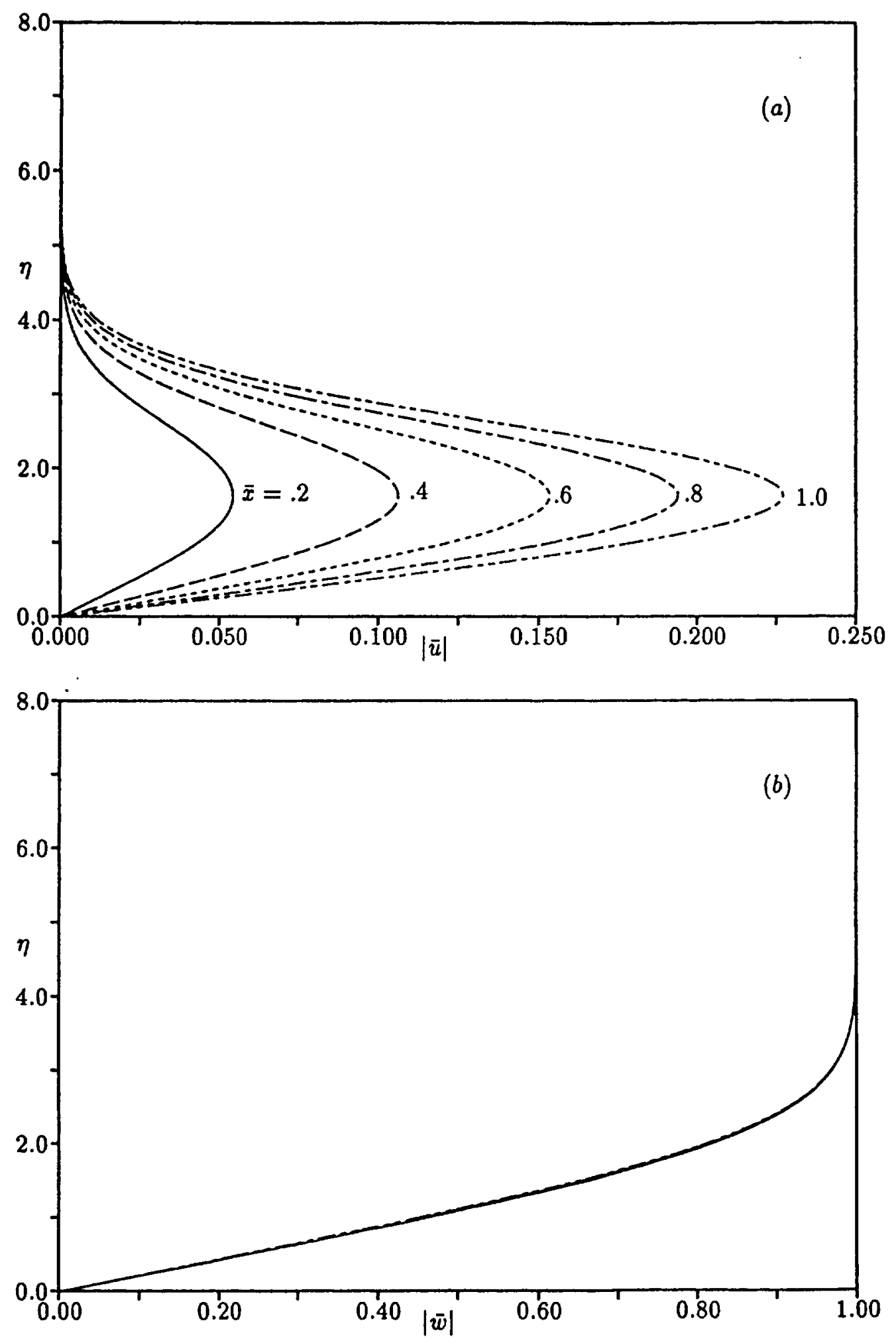

Figure 2: Profiles of (a) streamwise and (b) crossflow velocity perturbation from the boundary layer solution; $\bar{x}=0.2,0.4,0.6,0.8,1.0$. 

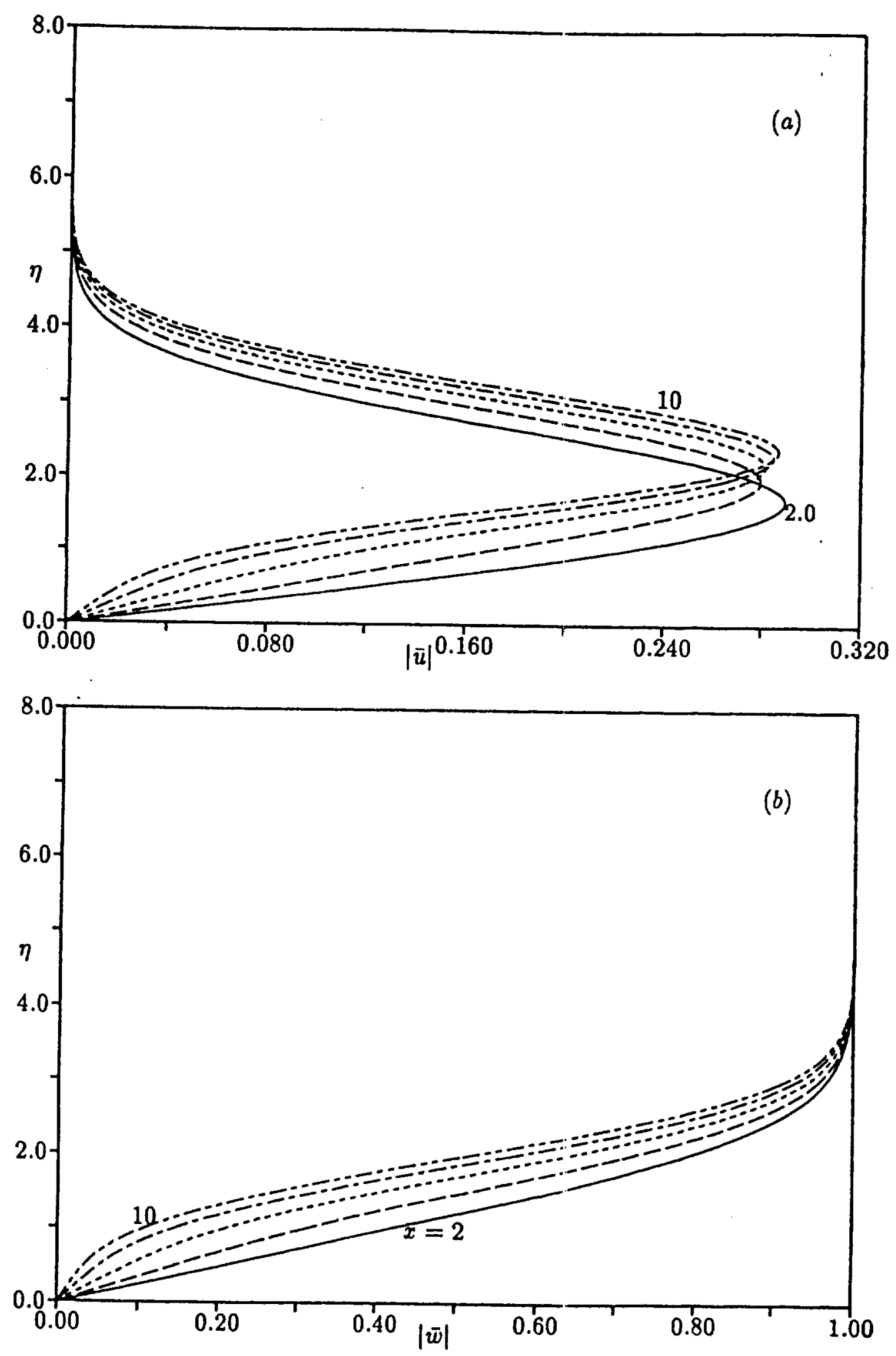

Figure 3: Profiles of (a) streamwise and (b) crossflow velocity perturbation from the boundary layer solution; $\bar{x}=2.0,4.0,6.0,8.0,10.0$. 

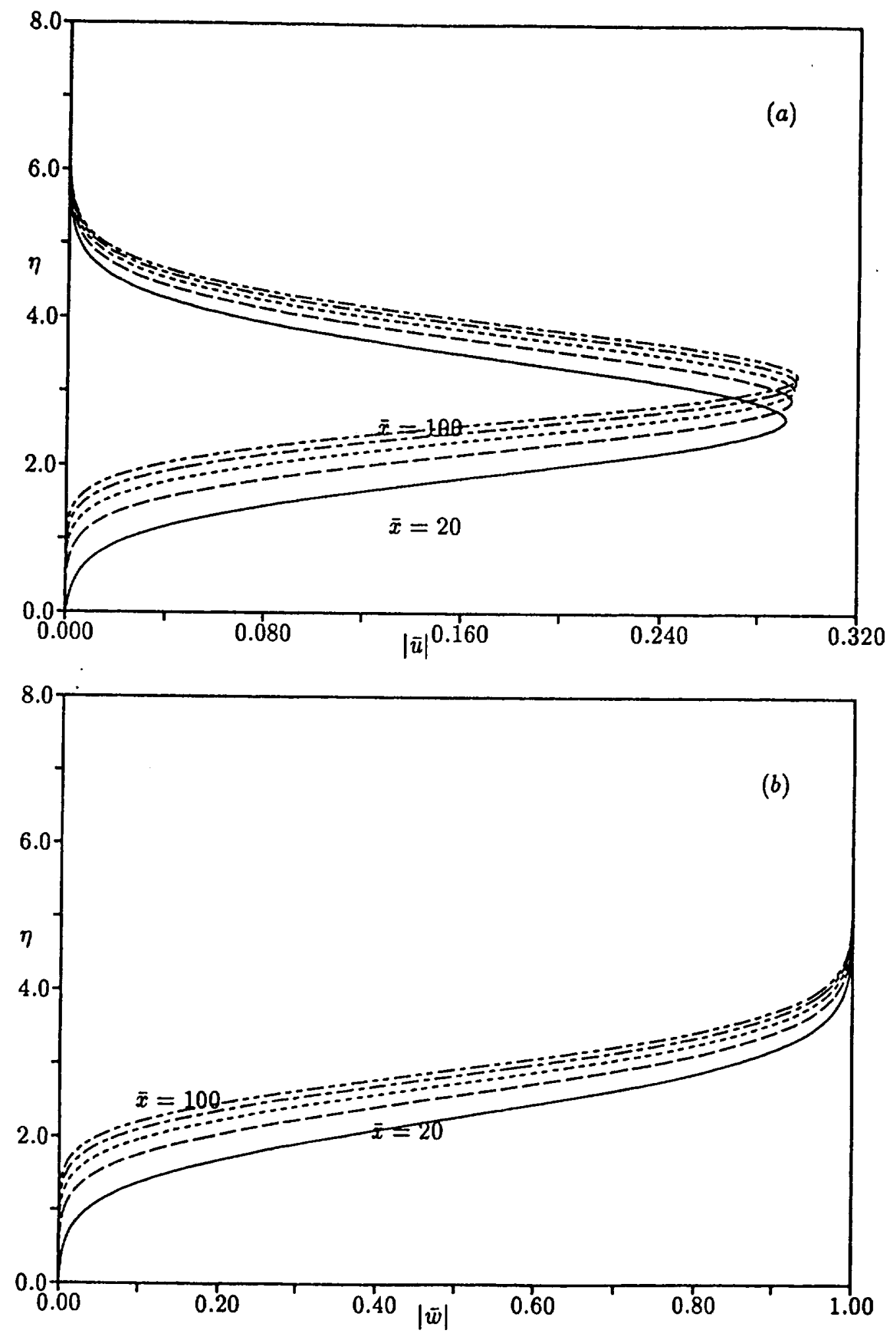

Figure 4: Profiles of (a) streamwise and (b) crossflow velocity perturbation from the boundary layer solution; $\bar{x}=20.0,40.0,60.0,80.0,100$. 

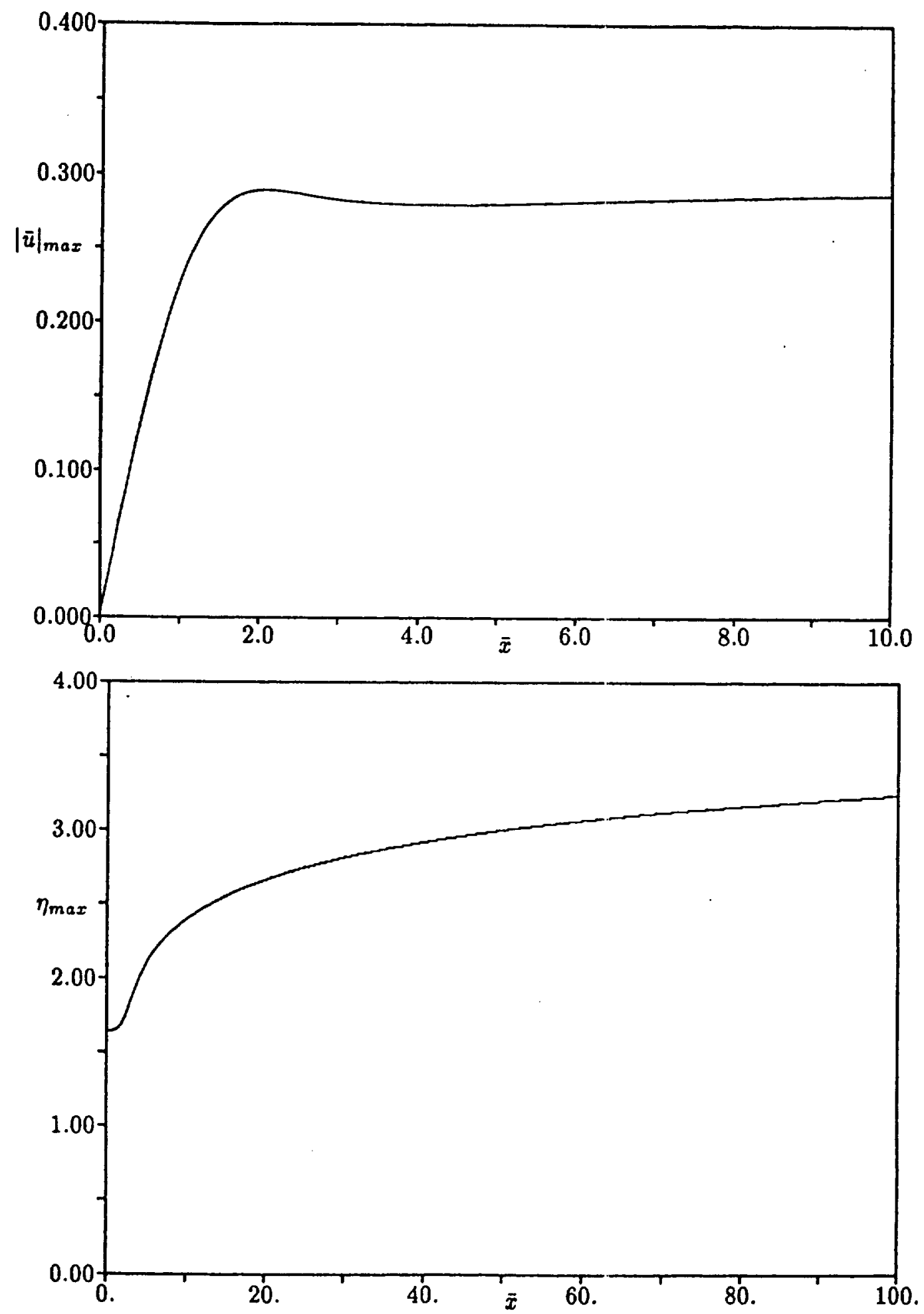

Figure 5: Downstream evolution of the peak of the streamwise velocity perturbation magnitude (a) $|\bar{u}|_{\max }$ (b) $\eta_{\max }$ 

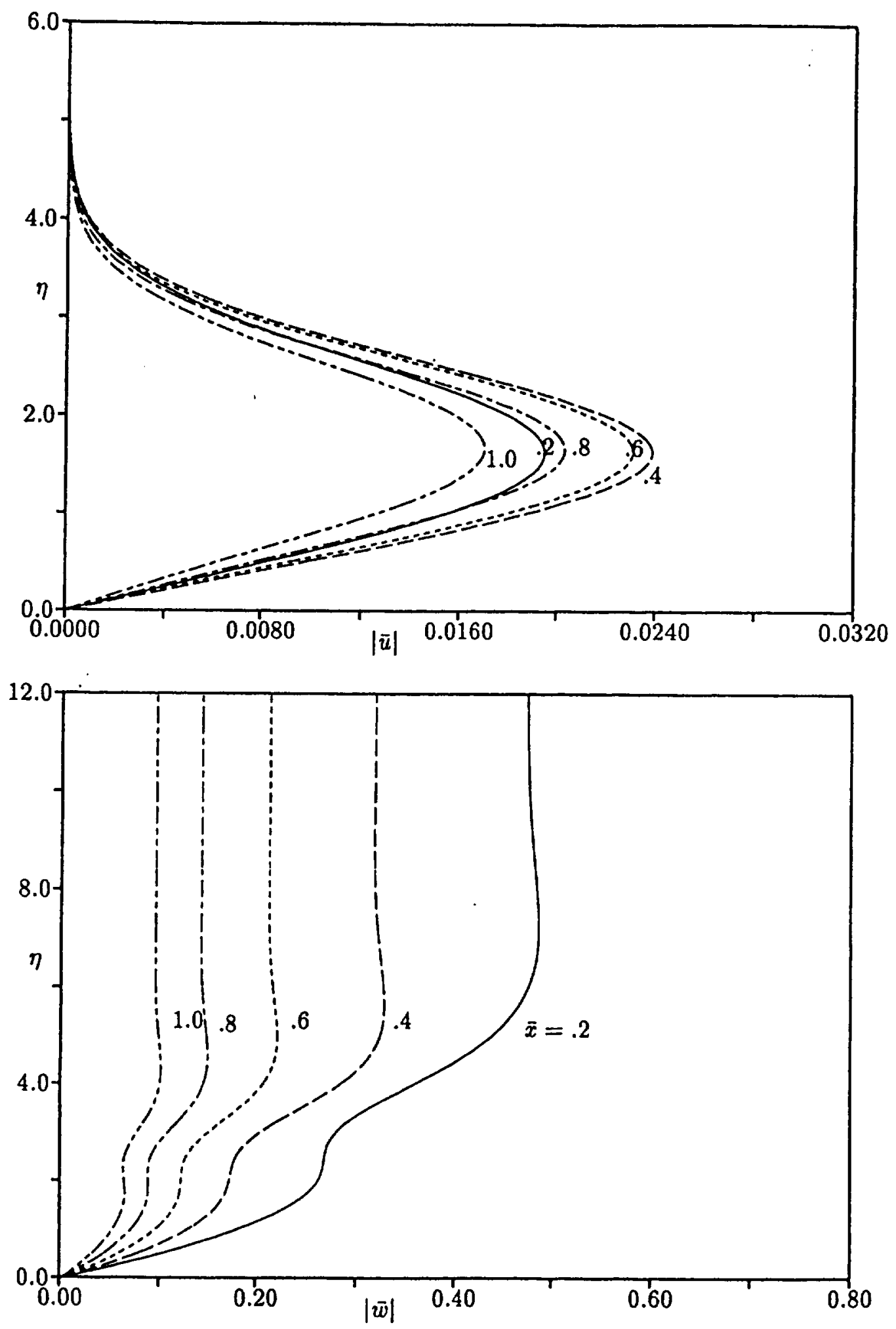

Figure 6: Profiles of (a) streamwise and (b) crossflow perturbation velocity for $\kappa=1, \kappa_{2}=$ -1 , at $\bar{x}=0.2,0.4,0.6,0.8,1.0$. 

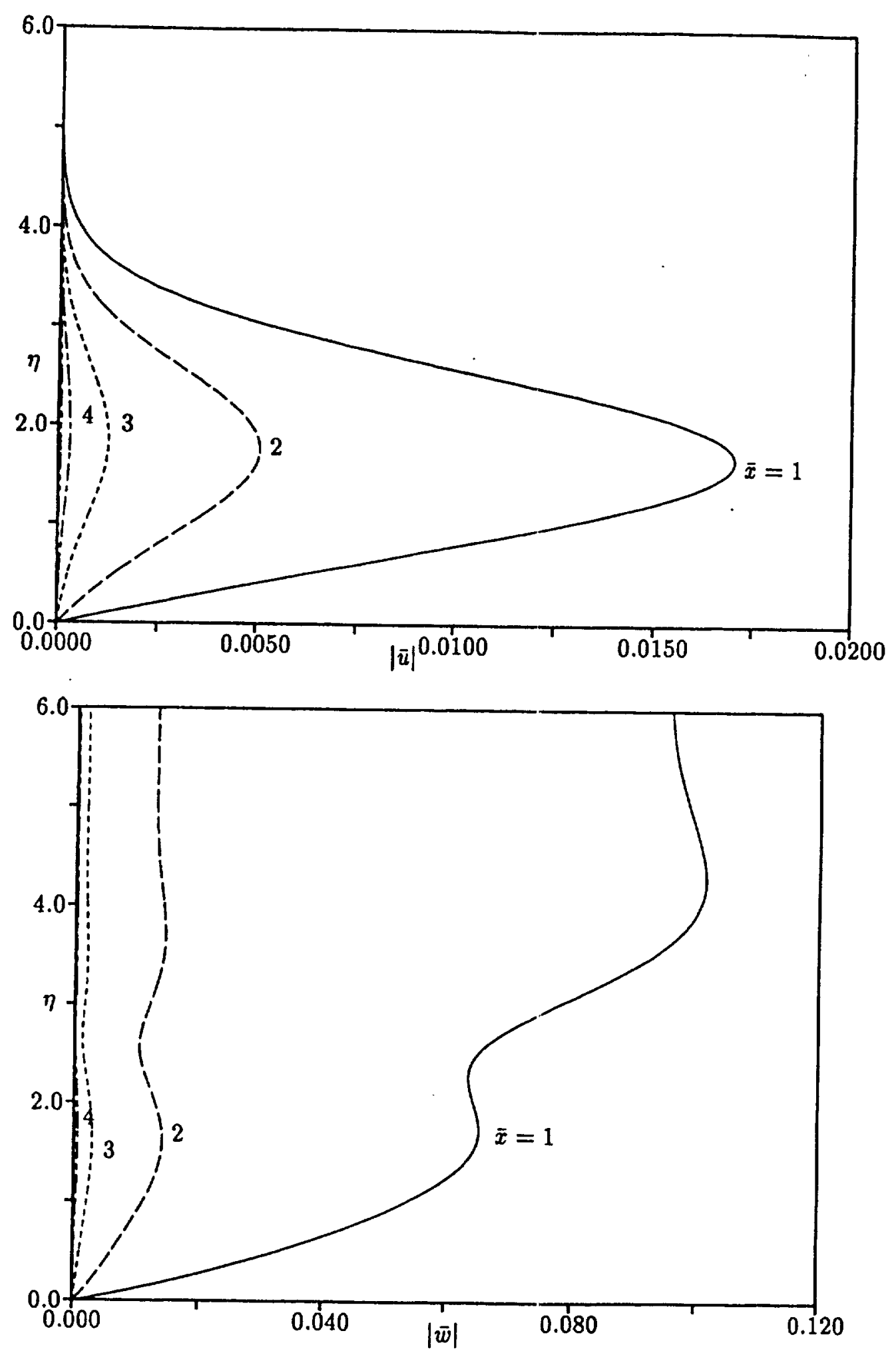

Figure 7: Profiles of (a) streamwise and (b) crossflow perturbation velocity for $\kappa=1, \kappa_{2}=$ -1 , at $\bar{x}=1.0,2.0,3.0,4.0$. 


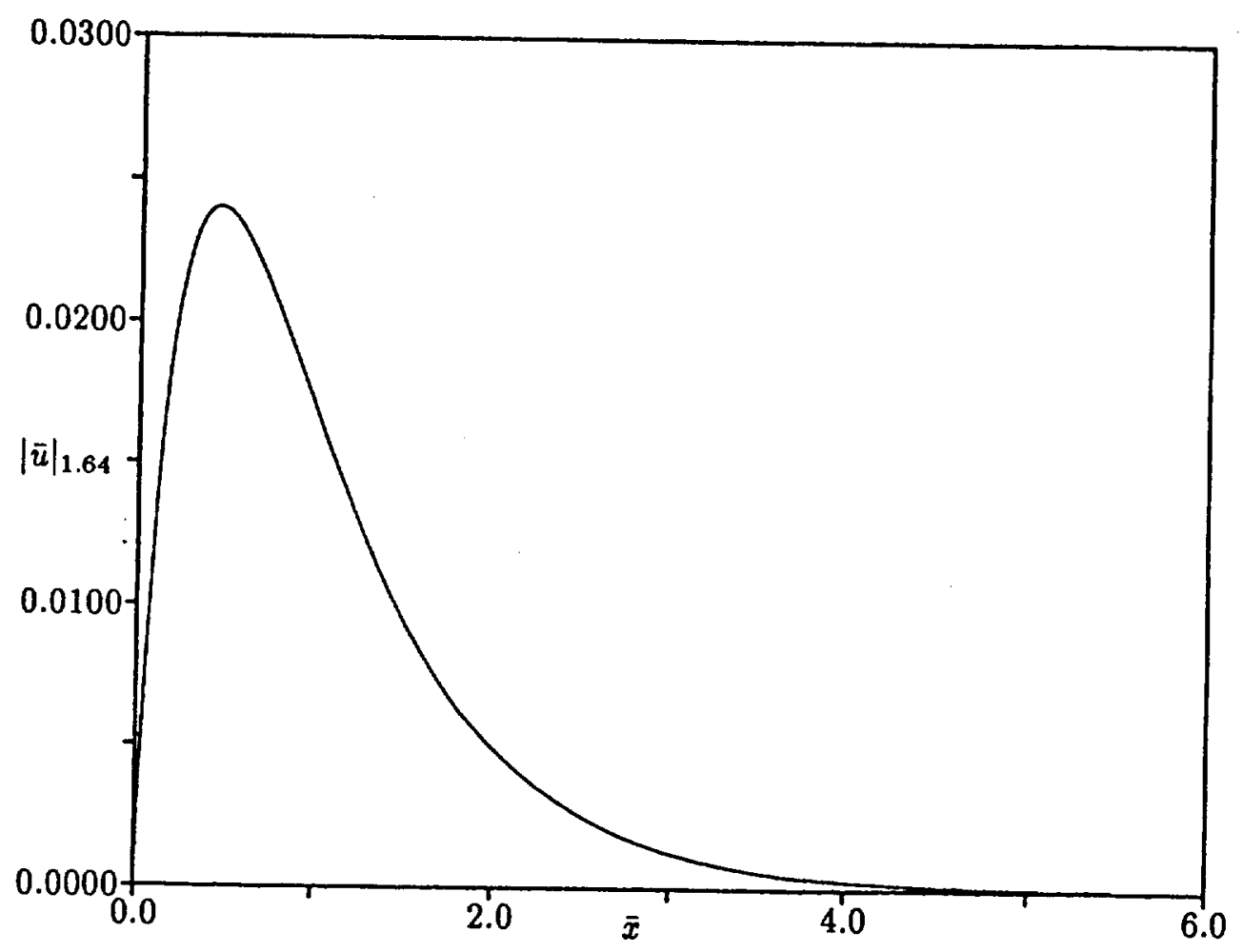

Figure 8: Evolution of the boundary region streamwise velocity perturbation magnitude at $\eta=1.64$ for $\kappa=1, \kappa_{2}=-1$. 

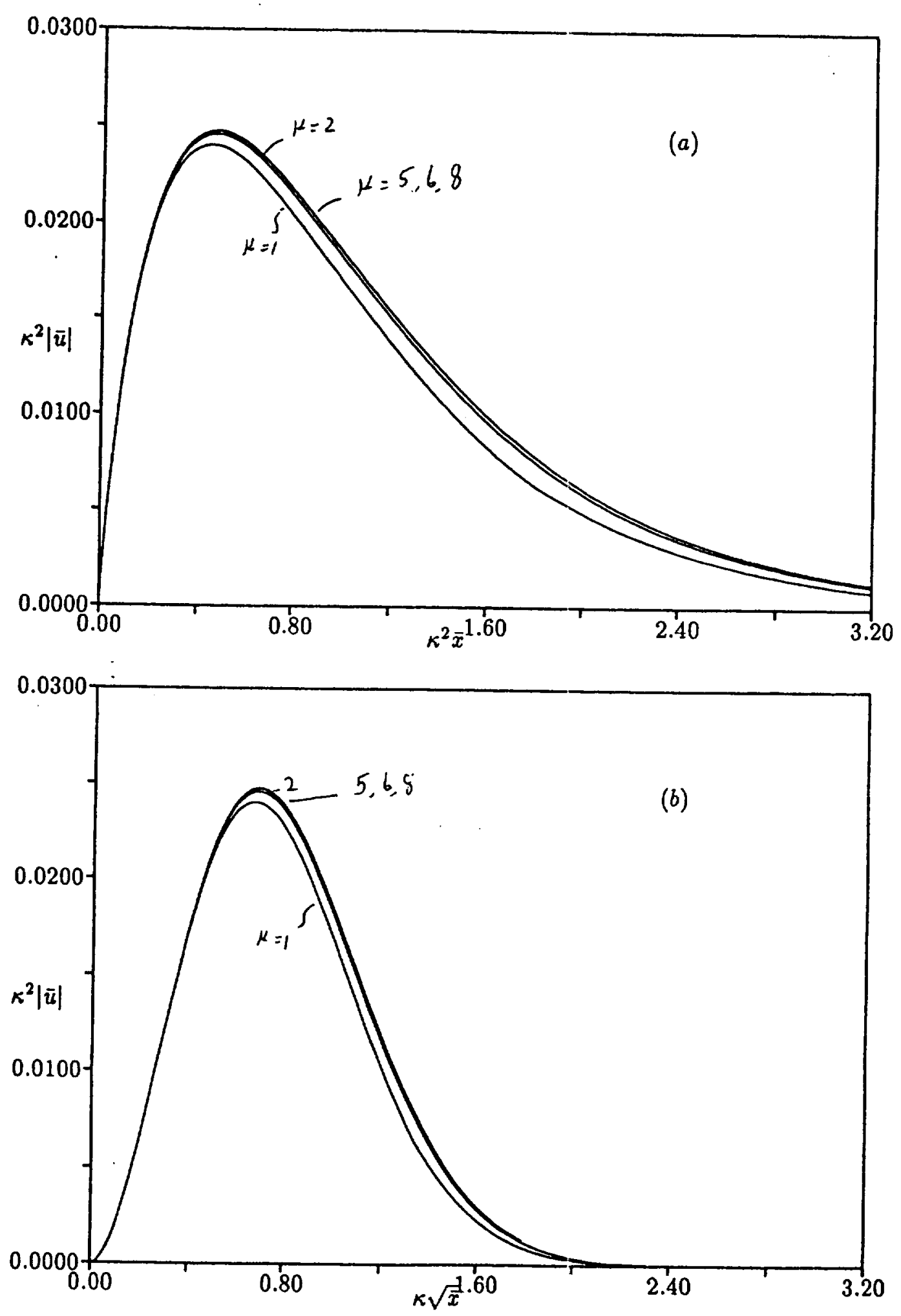

Figure 9: Plot of $\kappa^{2}|\bar{u}|$ vs. (a) $\kappa^{2} \bar{x}$ and (b) $\kappa \sqrt{\bar{x}}$ for various values of $\kappa=-\kappa_{2} \geqslant 1$. 


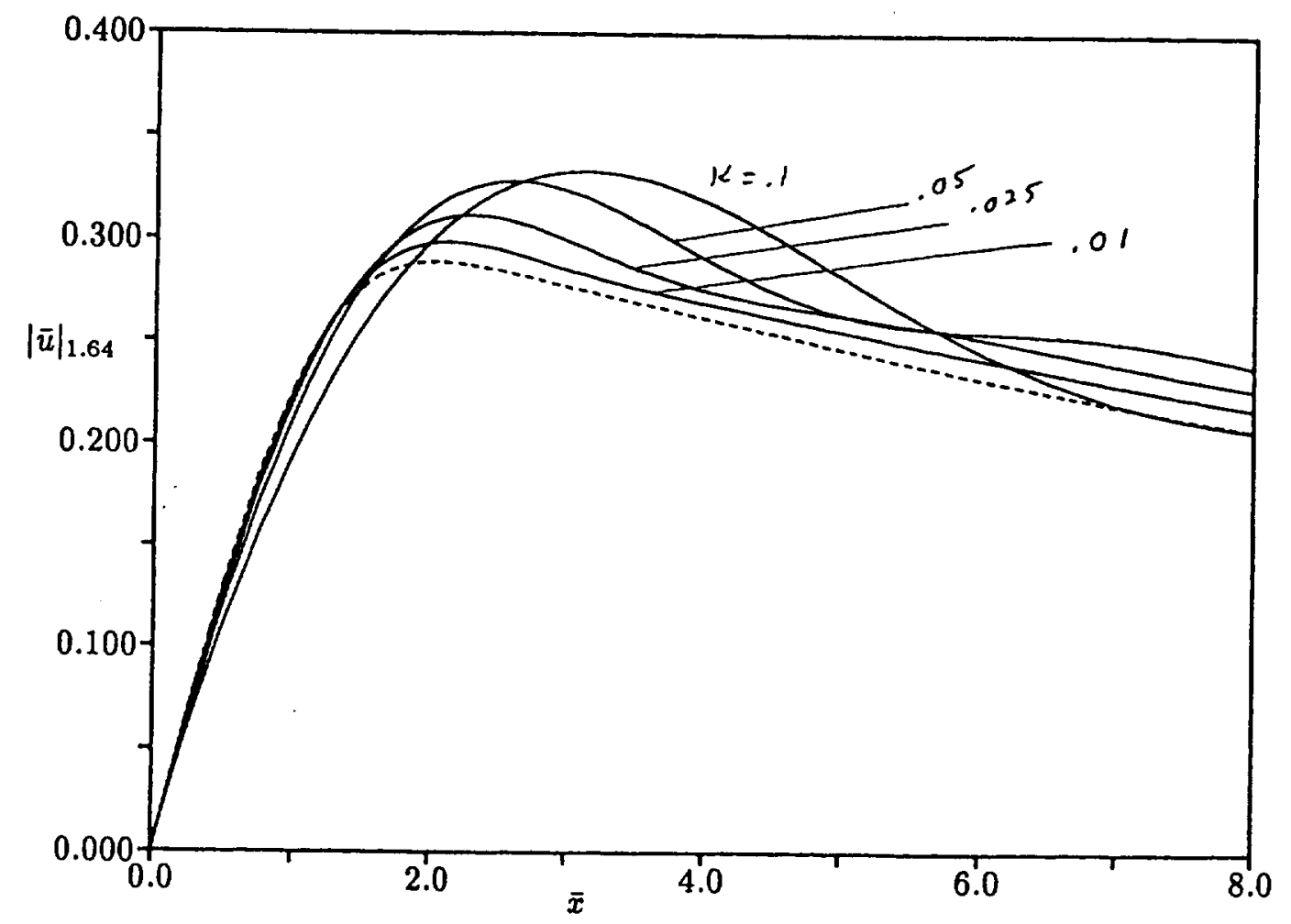

Figure 10: Curves of $|\bar{u}|$ at $\eta=1.64$ vs. $\bar{x}$ for various $\kappa \leqslant 0.1$ with $\kappa_{2}=-\kappa$ from the boundary region equations. The dashed curve is the corresponding boundary layer solution. 


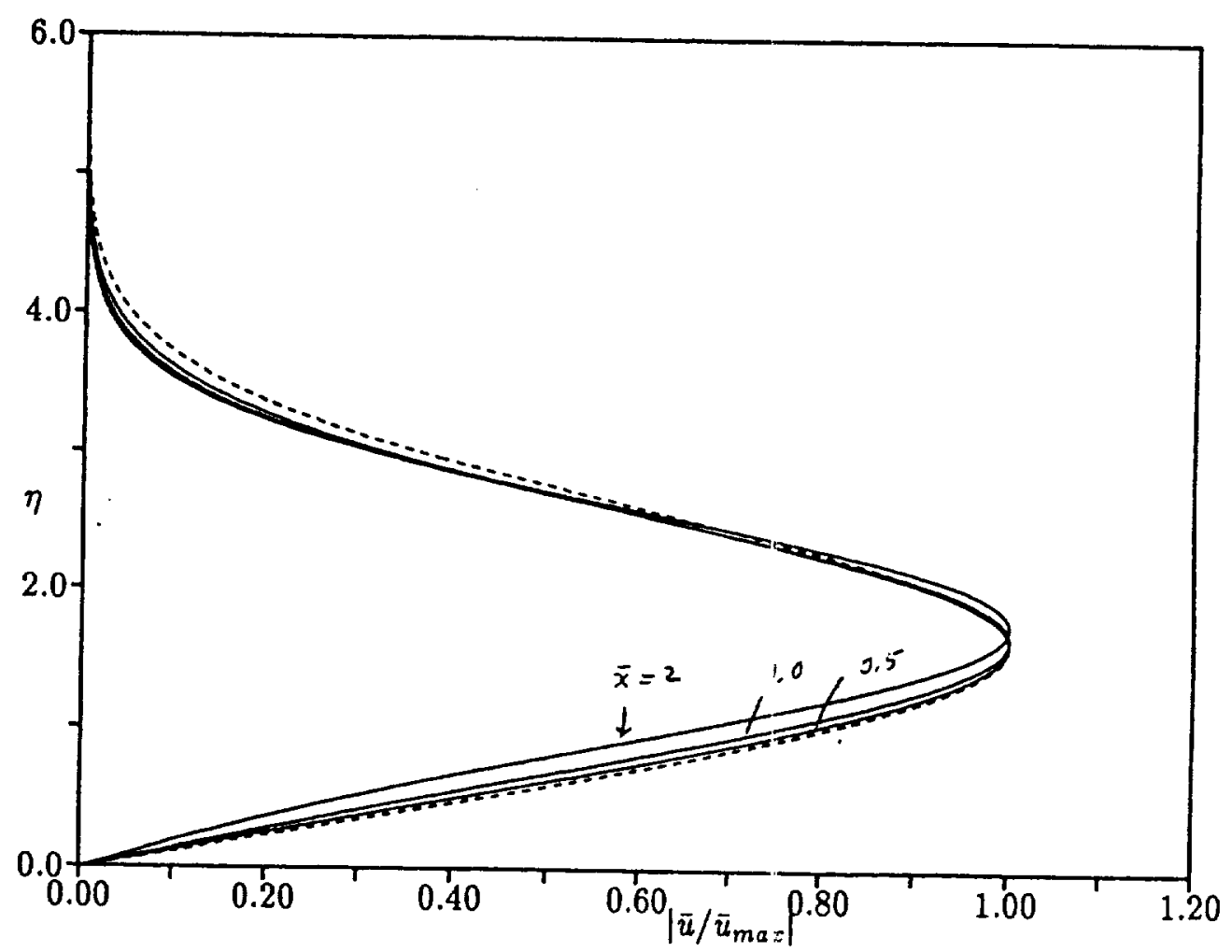

Figure 11: Profiles of $\left|\bar{u} / \bar{u}_{\max }\right|$ at indicated $\bar{x}, \kappa=1$ ard $\kappa_{2}=-1$. The dotted curve corresponds to $\eta F^{\prime \prime} /\left(\eta F^{\prime \prime}\right)_{1.64}$. 

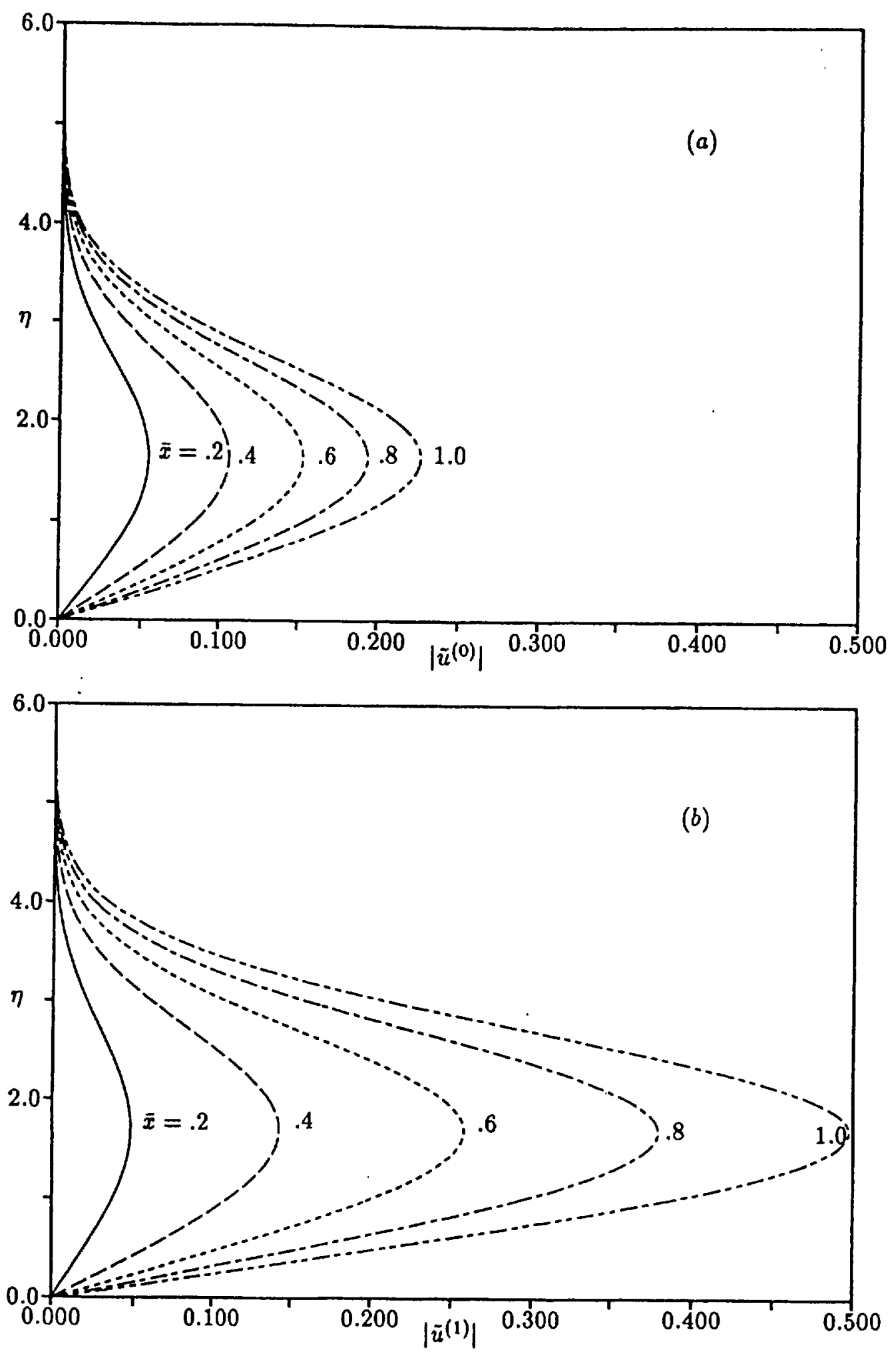

Figure 12: Plots of (a) leading order (boundary layer solution) and (b) first order streamwise velocity profiles in longwavelength limit for $\bar{x}=0.2,0.4,0.6,0.8,1.0$. 

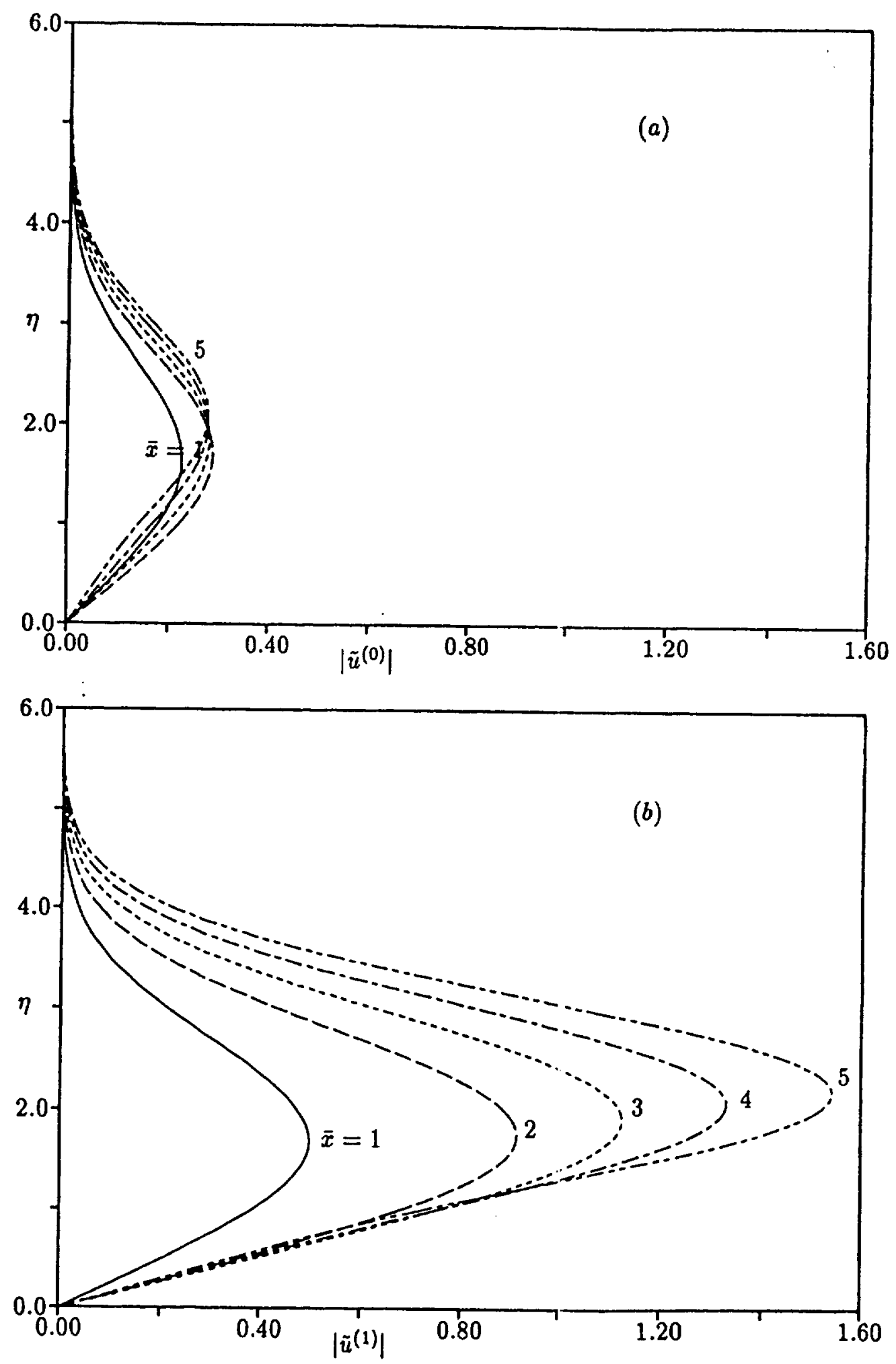

Figure 13: Plots of (a) leading order (boundary layer solution) and (b) first order streamwise velocity profiles in longwavelength limit for $\bar{x}=1.0,2.0,3.3,4.0,5.0$. 


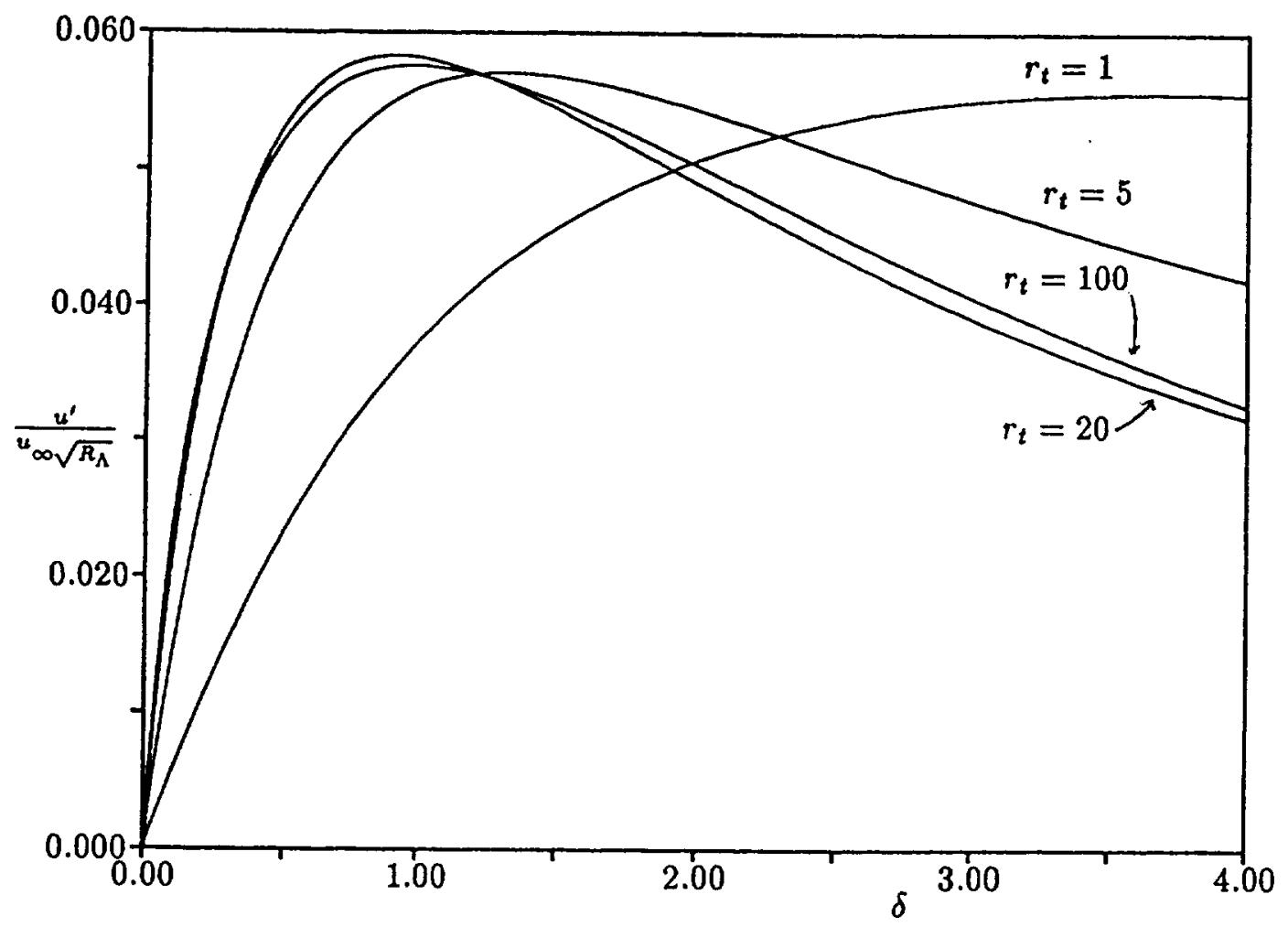

Figure 14: Normalized root mean square of the streamwise velocity fluctuations in the boundary layer for isotropic free stream turbulence at various turbulent Reynolds numbers. 

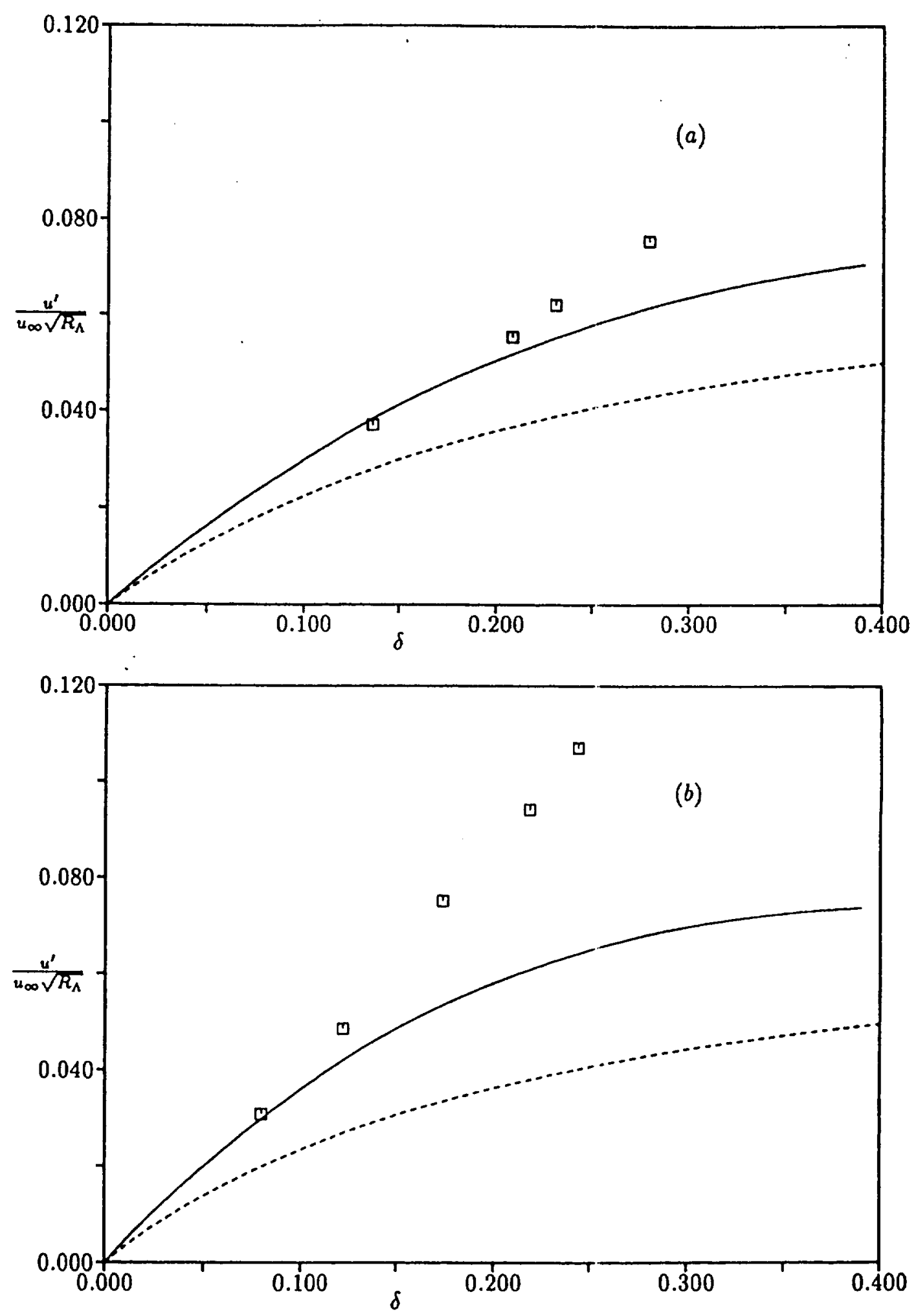

Figure 15: Comparison of theoretical results with experimental data of Westin et al (1994). Dashed curves are for isotropic turbulence, solid curves for the strongly anisotropic form (7.19). (a) $T u=1.35 \%, R_{\Lambda}=2120, r_{t}=29 ; k_{t}=1.75$ (b) $T u=1.5 \%, R_{\Lambda}=4240, r_{t}=64$ $; k_{t}=2.2$. 

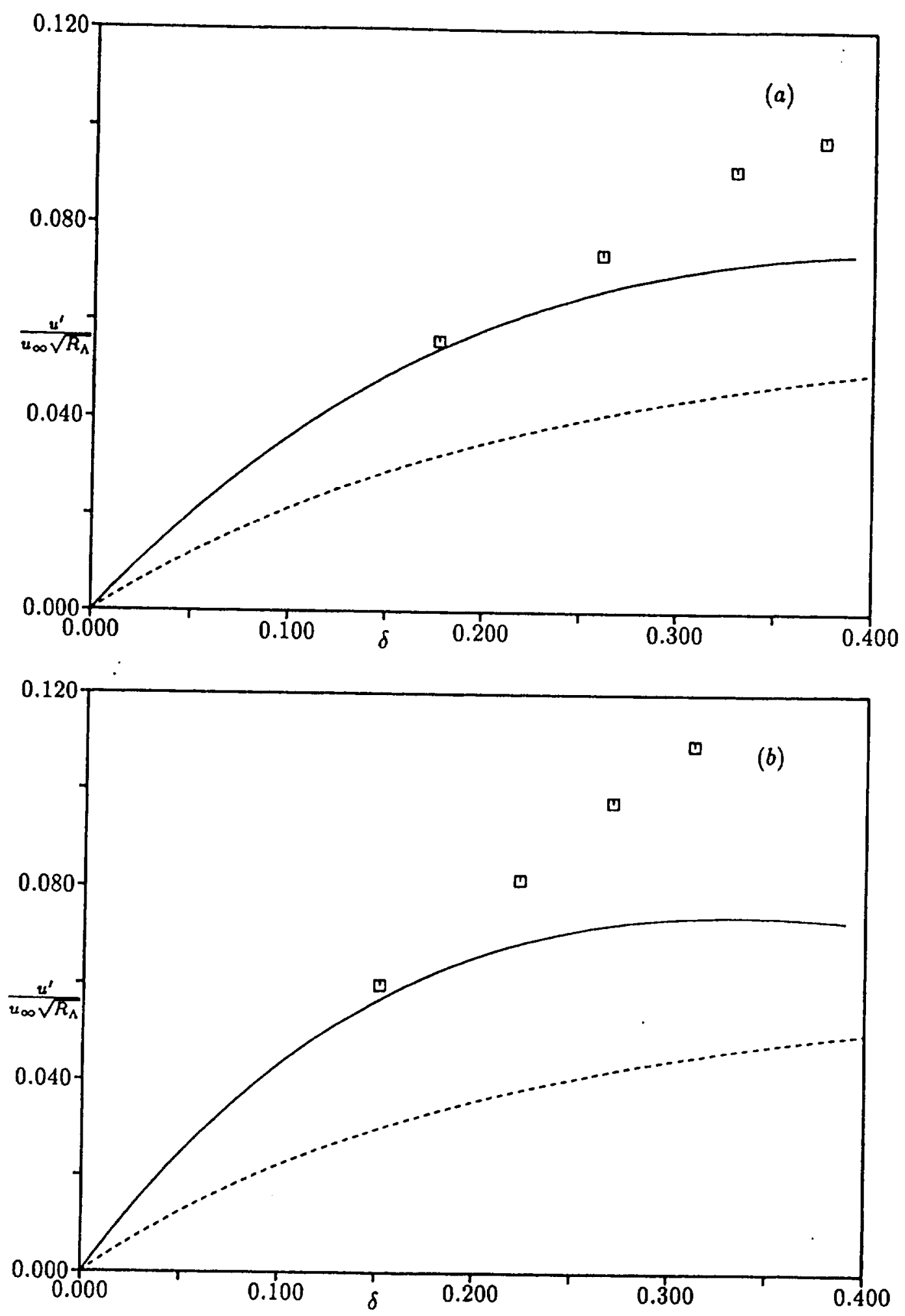

Figure 16: Comparison of theoretical results with experimental data of Roach \& Brierley (1992). Dashed curves are for isotropic free stream turbulence, solid curves for the strongly anisotropic form (7.19). (a) SMRLO, $T u=0.8 \%, R_{\Lambda}=2400, r_{t}=19 ; k_{t}=2.2$ (b) SMRHI, $T u=0.8 \%, R_{\Lambda}=3200, r_{t}=26 ; k_{t}=2.8$. 

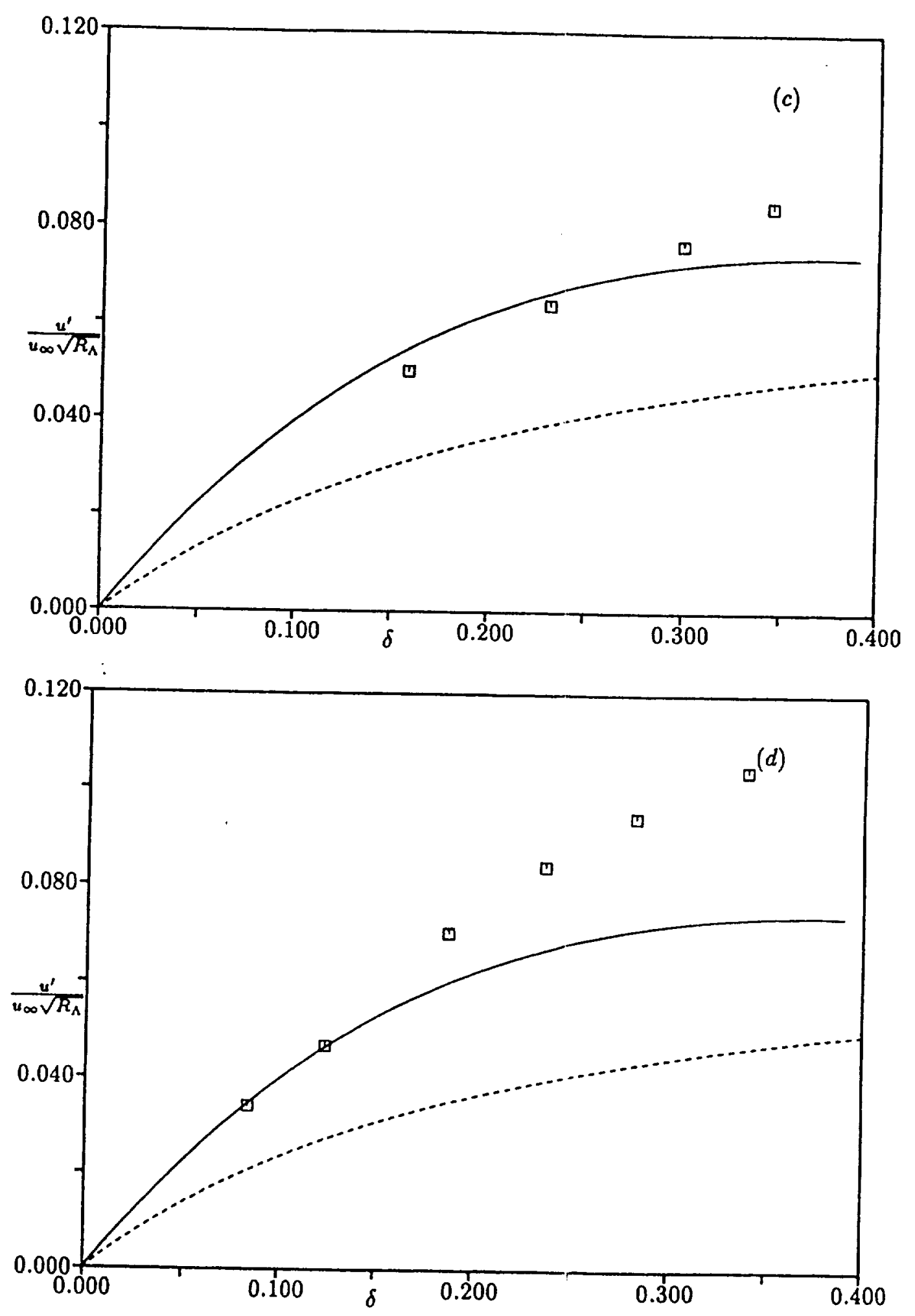

Figure 16: Comparison of theoretical results with experinental data of Roach \& Brierley (1992). Dashed curves are for isotropic free stream turtulence, solid curves for strongly anisotropic form (7.19). (c) PRLO, $T u=3.0 \%, R_{\Lambda}=1200, r_{t}=36 ; k_{t}=2.5$ (d) PRHI, $T u=3.0 \%, R_{\mathrm{A}}=2100, r_{t}=63 ; k_{t}=2.5$. 